\title{
Vigas de concreto armado reforçadas à flexão com barras de aço $e$ FRP no cobrimento do concreto
}

Este trabalho tem por objetivo apresentar um método teórico de previsão do momento fletor e da flecha de ruptura de vigas de concreto armado reforçadas à flexão com o uso da técnica da inserção de barras no cobrimento do concreto. Esta técnica é conhecida como a técnica NSM (Near Surface Mounted). No presente trabalho foram utilizadas barras de polímero reforçado com fibras (FRP). Os resultados teóricos foram comparados com os resultados experimentais disponíveis na literatura. Todas as vigas de concreto armado foram submetidas à flexão em quatro pontos. Os parâmetros considerados no método proposto foram as dimensões e taxas de armaduras internas das vigas, a resistência do concreto à compressão, a taxa e o tipo da armadura de reforço, o tipo de cola e o comprimento colado da barra de FRP. Foram analisadas vigas armadas com barras de CFRP, GFRP e aço. Os resultados teóricos obtidos apresentam uma boa aproximação com os resultados experimentais, indicando que a teoria da flexão simples pode ser utilizada para obtenção de valores razoáveis e a favor da segurança de momento fletor e flecha de ruptura de vigas retangulares reforçadas à flexão pela técnica NSM.

Palavras-chave: Reforço; Viga de Concreto Armado; Polímero Reforçado com Fibras; Inserção no Cobrimento do Concreto.

\section{Reinforced concrete beams reinforced with steel bars and FRP in concrete covering}

\begin{abstract}
This paper aims to present a theoretical method of prediction the maximum bending moment carrying capacity and the maximum mid-span deflections of reinforced concrete beams strengthened with fiber reinforced plastic (FRP) bars in the near-surface-mounted (NSM) technique. The theoretical results were compared with experimental results available in the literature. The reinforced concrete beams were submitted to four-point bending test. The theoretical parameters considered were the dimensions and internal reinforcement rate of the beams, the concrete compression strength, the type and rate of the externa reinforcement, the glue type and the bond length of the FRP bar. CFRP, GFRP and steel reinforced concrete beams were analyzed. The theoretical results presented good agreement with the experimental results, showing that the simple bending theory can be used to obtain bending moment carrying capacity and the mid-span deflections of reinforced rectangular concrete beams strengthened by the NSM technique.
\end{abstract}

Keywords: Strengthening; Reinforced Concrete Beam; Fiber Reinforced Polymer; Near Surface Mounted.

Topic: Sustentabilidade nos Transportes

Reviewed anonymously in the process of blind peer.
Received: $10 / 02 / 2018$

Approved: $24 / 03 / 2018$
Renan Henrique de Melo

Instituto Militar de Engenharia, Brasil

http://lattes.cnpq.br/2049710504200235

renanime@hotmail.com

Ana Maria Abreu Jorge Teixeira

Instituto Militar de Engenharia, Brasil

http://lattes.cnpq.br/1454254574734029

anam@ime.eb.br

Luiz Antonio Vieira Carneiro

Instituto Militar de Engenharia, Brasil

http://lattes.cnpq.br/2431901858566512

carneiro@ime.eb.br

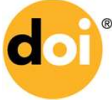

DOI: 10.6008/CBPC2179-6858.2018.003.0019
Referencing this:

MELO, R. H.; TEIXEIRA, A. M. A. J.; CARNEIRO, L. A. V.. Vigas de concreto armado reforçadas à flexão com barras de aço e FRP no cobrimento do concreto. Revista Ibero Americana de Ciências Ambientais, v.9, n.3, p.234-250, 2018. DOI: http://doi.org/10.6008/CBPC2179-6858.2018.003.0019 


\section{INTRODUÇÃO}

As estruturas de concreto armado são amplamente adotadas em edificações pelo mundo, devido à simplicidade para execução das mesmas. No Brasil existem 6.612 viadutos e pontes, aproximadamente, de concreto armado, um patrimônio avaliado em cerca de 13 bilhões de reais. Essas estruturas costumam estar submetidas a condições ambientais adversas, como altas temperaturas e umidade, cloretos oriundos do mar, sulfatos e chuvas ácidas, dentre outras. Estes fatores podem acarretar danos às estruturas, como a corrosão do aço, que devem ser reparados. Erros de construção ou de projeto e possíveis mudanças de aplicação da obra de arte também podem demandar reforço das estruturas de concreto.

Há vários materiais e técnicas voltados para o reparo ou reforço de estruturas de concreto armado, sendo a sua escolha decorrente, principalmente, das causas e extensões dos problemas, da disponibilidade dos materiais de reforço, da presença de pessoal especializado e de equipamentos adequados, da viabilidade técnico-econômica e da agressividade do meio ambiente.

As técnicas de reparo ou reforço mais usuais são a injeção de cimento ou resina epóxi nas fissuras, a realcalinização do concreto com aplicação superficial de argamassas ricas em cimento ou de concreto projetado, a adição de cabos de protensão externos e a colagem de chapas de aço (BEBER et al., 2000). A engenharia de materiais avançou muito nas últimas décadas em relação ao tipo de material utilizado nos serviços de reparo e reforço de estruturas, objetivando a redução do peso do material aplicado e o aumento de sua resistência e durabilidade.

Na década de 90, os materiais compósitos de resinas e fibras ou FRP (fiber reinforced polymers) começaram a ser mais pesquisados, com aplicações nas engenharias aeroespacial, aeronáutica, marítima, militar, automobilística, esportiva e de construção. Esses materiais apresentam elevada resistência mecânica, baixo peso específico e são aplicados em lâminas de pequena espessura, por isso, pouco modificam as dimensões dos elementos estruturais a serem reparados ou reforçados.

As técnicas de reparo e reforço de estruturas com o FRP utilizadas são a técnica colagem externa ou EB (Externally Bonded) e a técnica de embutimento no cobrimento do concreto ou NSM (Near Surface Mounted). No último caso, como o material fica embutido em sulcos no concreto, o mesmo fica protegido e a dimensão da estrutura praticamente não se altera. Essas são duas vantagens da técnica NSM sobre a EB, ocorrendo menor exposição a vandalismos e menor suscetibilidade à temperatura e danos.

Diversas pesquisas têm investigado o reforço à flexão de vigas de concreto por meio da técnica NSM, tendo esta técnica apresentado melhor desempenho do que a técnica EB. Não existe uma normatização brasileira para a técnica NSM, sendo necessária a utilização de normas estrangeiras quando da sua utilização. Existem empresas brasileiras que trabalham com a produção de FRP, porém em seus catálogos não apresentam dados de utilização do material para este fim. A técnica NSM é pouco utilizada no mercado brasileiro, não tendo sido encontradas publicações nacionais, que tratem de reparo ou reforço à flexão com o uso desta técnica. 
Este trabalho tem como finalidade apresentar um método teórico de previsão do momento fletor e da flecha de ruptura de vigas de concreto armado reforçadas à flexão com barras de FRP com a técnica NSM. Os resultados teóricos foram comparados com os resultados experimentais apresentados em pesquisas publicadas a partir do ano de 2000.

\section{REVISÃO TEÓRICA}

\section{Polímeros reforçados com fibras (FRP)}

Materiais compósitos ou polímeros reforçados com fibras são materiais cuja estrutura é constituída por uma combinação de dois ou mais elementos não solúveis entre si. Um dos seus elementos é chamado de reforço e outro de matriz polimérica, que envolve o primeiro. O reforço é constituído por fibras, que podem ser encontradas em diversas formas como: tecidos, barras, fitas e laminados. As principais fibras adotadas em FRP são de vidro, carbono, aramida e basalto. As matrizes poliméricas mais adotadas são de poliéster, epóxi, fenólica e éster vinílica.

As fibras têm o papel de conferir rigidez e resistência ao material compósito. A matriz protege as fibras da umidade e de ambientes alcalinos (fibra de vidro) e ácidos (fibras de aramida), evita a flambagem das fibras e possibilita a transferência de carga entre as mesmas, tendo em sua composição adições tais como aditivos e materiais de enchimento (fillers), que modificam e melhoram suas propriedades. A Figura 1 mostra barras e laminados de FRP comumente empregados no reforço de lajes e vigas de concreto. Essas barras e laminados são fabricados pelo processo de pultrusão, que consiste em 'puxar' as fibras embebidas na resina matriz através de um molde. O aquecimento do molde, que tem a forma da seção transversal do perfil desejado, provoca a polimerização da resina durante a passagem no seu interior. A Figura 2 ilustra o processo de pultrusão.

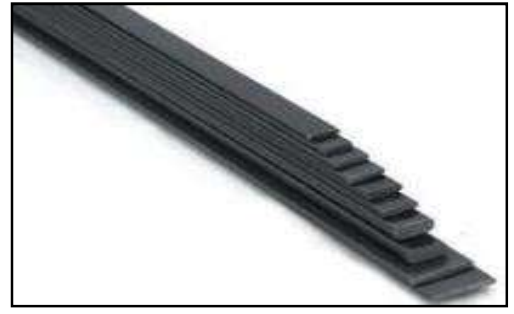

(a)

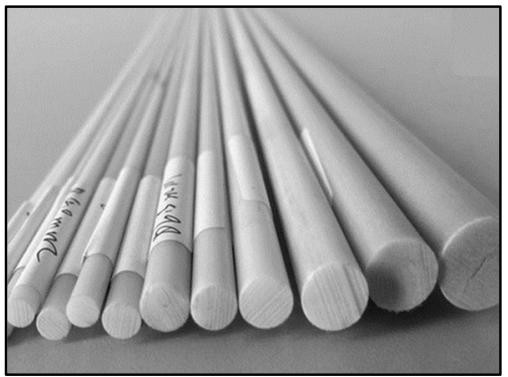

(c)

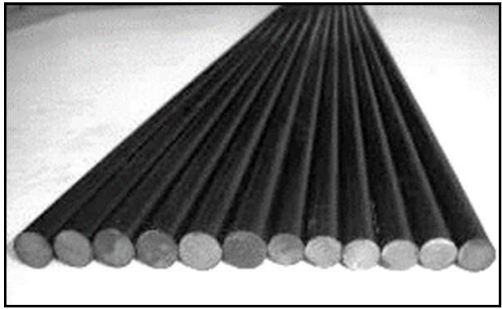

(b)

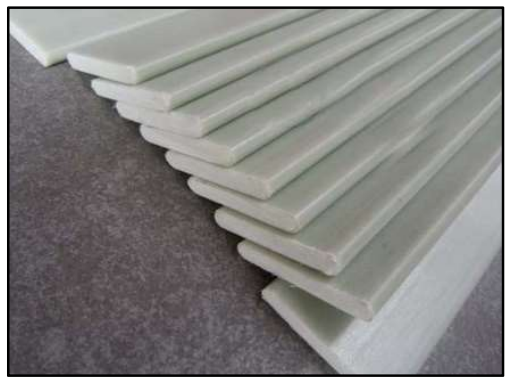

(d)

Figura 1: FRP comumente utilizados no reforço de lajes e vigas de concreto armado: (a) lâmina de CFRP (carbonfiberproducts), (b) barra de CFRP (FRP Products), (c) barra de GFRP (Alibaba.com), (d) barra chata de GFRP

(Aliexpress.com). 


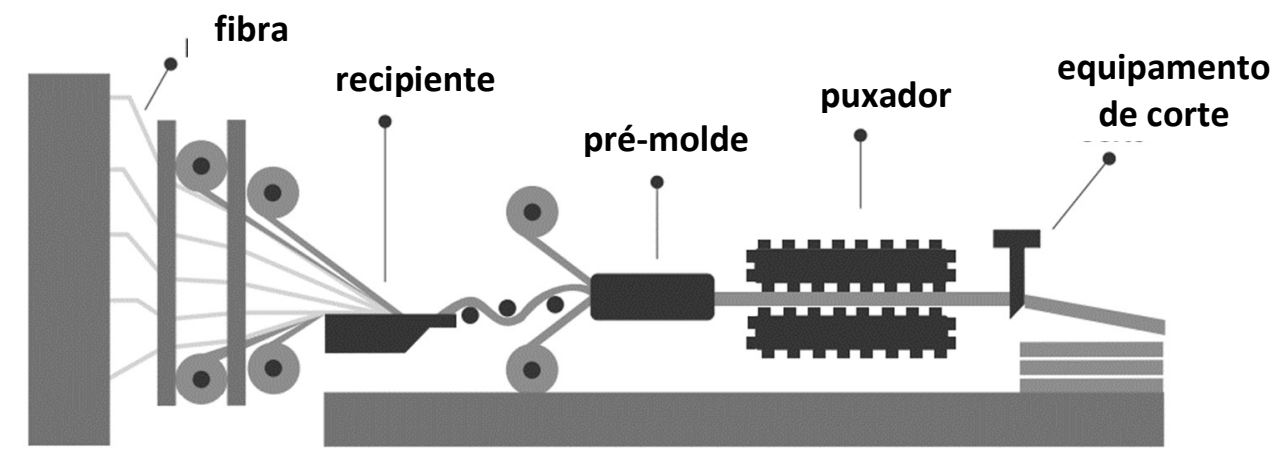

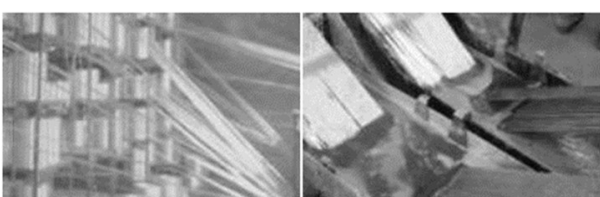

fibra recipiente

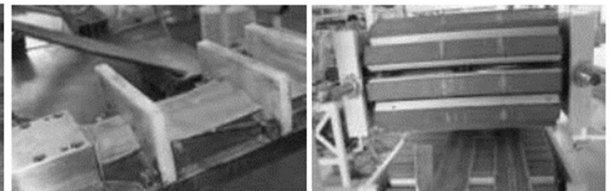

pré-molde puxador

Figura 2: Processo da pultrusão utilizado na fabricação das barras de GFRP (STRATUS).

\section{Dados experimentais}

Do levantamento feito na literatura sobre as pesquisas englobando o reforço à flexão de vigas de concreto armado pela técnica NSM, foram encontrados 24 trabalhos correspondendo ao ensaio de flexão em quatro pontos de 57 vigas de seção transversal retangular, com armadura interna formada por barras de aço. Os estudos abordaram o uso de barras e fitas rígidas de CFRP, barras de GFRP e barras de aço como materiais de reforço.

Na Tabela 1 são apresentados, de forma resumida e por ordem cronológica, os estudos encontrados na literatura sobre o comportamento de vigas de concreto armado reforçadas à flexão pelo método NSM e os resultados obtidos pelos autores. As vigas desses estudos possuem largura (b) variando de $100 \mathrm{~mm}$ a 250 $\mathrm{mm}$, altura (h) de $150 \mathrm{~mm}$ a $350 \mathrm{~mm}$, vão livre (L) de $900 \mathrm{~mm}$ a $3600 \mathrm{~mm}$, taxa de armadura longitudinal interna de tração $\left(\rho_{s}\right)$ de $0,23 \%$ a $1,26 \%$, taxa de armadura transversal $\left(\rho_{w}\right)$ de $0,24 \%$ a $1,31 \%$ e taxa de reforço ( $\left.\rho_{f}\right)$ de $0,06 \%$ a $0,75 \%$.

Os valores de resistência média do concreto à compressão $\left(f_{c}\right)$ das vigas situa-se entre $20 \mathrm{MPa}$ e 65 MPa e as deformações específicas do reforço obtidas durante os ensaios variaram de $0,6 \%$ a $1,75 \%$. Os modos de ruptura das vigas indicadas na Tabela 1 estão ilustrados na Figura 3. Verifica-se que os modos de ruptura são influenciados por vários aspectos, como o comprimento colado, as dimensões da seção do sulco, diâmetro, quantidade e tipo do material do reforço e tipo de cola. Os tipos de ruptura mais comuns apresentados nas pesquisas foram: falha na interface concreto/epóxi (CE), destacamento do concreto (DC), divisão do concreto em torno do compósito (DCTC) e ruptura do compósito (RC).

Da análise dos resultados dos trabalhos, foram feitas as seguintes constatações: - $O$ tipo de material de reforço influencia significativamente o comportamento da viga reforçada, em termos de sua carga de ruptura, rigidez e ductilidade. 
Tabela 1: Quadro resumo dos dados das vigas ensaiadas por diferentes autores.

\begin{tabular}{|c|c|c|c|c|c|c|c|c|c|c|c|c|c|c|c|c|c|c|c|}
\hline \multirow{3}{*}{ Autor } & \multicolumn{10}{|c|}{ Características geométricas e propriedades mecânicas das vigas } & \multicolumn{5}{|c|}{$\begin{array}{c}\text { Características geométricas e propriedades } \\
\text { mecânicas dos reforços NSM }\end{array}$} & \multirow{3}{*}{ Modo de Ruptura } & \multirow{3}{*}{$\begin{array}{l}\text { Mu,exp } \\
(\mathrm{kN} . \mathrm{m})\end{array}$} & \multirow{3}{*}{$\varepsilon_{\mathrm{t}, \mathrm{exp}}(\%)$} & \multirow{3}{*}{$\begin{array}{r}\Delta u, \exp \\
(\mathrm{mm})\end{array}$} \\
\hline & $\mathrm{h}$ & $b$ & $\mathrm{~L}$ & $a$ & d & $\mathrm{c}_{\mathrm{t}}$ & $f_{y}$ & $f_{c}$ & $\rho_{\mathrm{s}}$ & $\rho_{\mathrm{sw}}$ & Tipo de & $\Phi_{\mathrm{f}}$ & $\rho_{\mathrm{f}}$ & $f_{f u}$ & $E_{f}$ & & & & \\
\hline & $\mathrm{mm}$ & $\mathrm{mm}$ & $\mathrm{mm}$ & $\mathrm{mm}$ & $\mathrm{mm}$ & $\mathrm{mm}$ & $\mathrm{MPa}$ & $\mathrm{MPa}$ & $\%$ & $\%$ & Mat. & $\mathrm{mm}$ & $\%$ & $\mathrm{MPa}$ & Gpa & & & & \\
\hline \multirow{4}{*}{ BARROS e FORTES [8] } & 170 & \multirow{4}{*}{100} & \multirow{4}{*}{1500} & \multirow{4}{*}{500} & 141 & & \multirow{2}{*}{730} & \multirow{4}{*}{45} & $0,40 \%$ & \multirow{4}{*}{$0,57 \%$} & & & $0,08 \%$ & & & NR & 12,58 & 15,50 & 4,62 \\
\hline & 177 & & & & 148 & 20 & & & $0,57 \%$ & & Fita CFPP & $059 \times 145$ & $0,16 \%$ & 27305 & 1588 & & 23,73 & 10,60 & 4,22 \\
\hline & 175 & & & & 145,6 & 20 & 524.2 & & $0,39 \%$ & & rilde crne & (3) & $0,16 \%$ & & & DC & 19,63 & 12,80 & 4,72 \\
\hline & 180 & & & & 150 & & & & $1,01 \%$ & & & & $0,24 \%$ & & & & 20,48 & 12,80 & 4,34 \\
\hline & & & & & 141,5 & & 788 & & $0,23 \%$ & & & & $0,07 \%$ & & & & 11,99 & & 10,50 \\
\hline BARROS et al. [9] & 170 & 120 & 900 & 300 & 14075 & 20 & 627 & 50 & $0,39 \%$ & $0,59 \%$ & Fita CFRP & $9,6 \times 1,4$ & $0,14 \%$ & 2740 & 158,8 & $E L A-D C$ & 14,00 & $\mathrm{NI}$ & 6,00 \\
\hline & & & & & 140,15 & & 621 & & $0,59 \%$ & & & & $0,20 \%$ & & & & 14,49 & & 3,70 \\
\hline NORDIN E TÄLSTEN & 300 & 200 & 3600 & 1300 & 253 & 30 & 496 & 65 & $061 \%$ & $105 \%$ & Barm CFRP & $10 \times 10$ & $017 \%$ & 2800 & 160 & $B C$ & 79,95 & N11 & 50,00 \\
\hline$[10]$ & 300 & 200 & 3600 & 1300 & 253 & 30 & 496 & 65 & $0,61 \%$ & $1,05 \%$ & Ba rra CFRP & $10 \times 10$ & $0,1 / \%$ & 2800 & 250 & $\mathrm{KC}$ & 79,30 & NoI & 41,00 \\
\hline TENG et al. [11] & 300 & 150 & 3000 & 1200 & 256 & 30 & 503 & 44 & $0,59 \%$ & $0,67 \%$ & Fita CFRP & $16 \times 4$ & $0,15 \%$ & 2068 & 151 & DC & 59,88 & $\mathrm{NI}$ & $\mathrm{NI}$ \\
\hline WANG et al. [12] & 300 & 150 & 3000 & 1200 & 256 & 30 & 576,3 & 37,5 & $0,59 \%$ & $0,67 \%$ & Barra GFRP & 9,5 & $0,16 \%$ & 831 & 42,6 & RFEC - CE - RC & 48,90 & 17,50 & 58,00 \\
\hline & & & & & & & & 30 & & & & 6 & $0,14 \%$ & & & DCTC & 53,30 & & \\
\hline $\begin{array}{l}\text { AL-MAHMOUD et al. } \\
\text { [13] }\end{array}$ & 280 & 150 & 2800 & 800 & 243 & 25 & 600 & 30 & $0,62 \%$ & $0,25 \%$ & Ba rra CFRP & 12 & $0,28 \%$ & 1875 & 146 & $E C-D C$ & 65,40 & $\mathrm{NI}$ & $\mathrm{NI}$ \\
\hline & & & & & & & & 60 & & & & & & & & DCTC & 73,20 & & \\
\hline $\begin{array}{c}\text { AL-IVARIVIUUD etal. } \\
\text { [1, }\end{array}$ & 280 & 150 & 1800 & 800 & 248 & 20 & 600 & 30 & $0,61 \%$ & $0,25 \%$ & Ba rra CFRP & 6 & $0,14 \%$ & 1875 & 146 & DCTC & 53,30 & $\mathrm{NI}$ & $\mathrm{NI}$ \\
\hline CHOI et al. [15] & 300 & 150 & 3300 & 1500 & 245,65 & 40 & $\mathrm{~N} / \mathrm{A}$ & 50 & $1,09 \%$ & $0,56 \%$ & Ba rra CFRP & 7,9 & $0,19 \%$ & 2800 & 155 & $\mathrm{RC}$ & 93,07 & 12,41 & 86,35 \\
\hline SENA-CRUZ et al. [16] & 300 & 200 & 2000 & 900 & 269 & 20 & $N / A$ & 50 & $0,44 \%$ & $0,28 \%$ & Fita CFRP & $15 \times 1,41$ & $0,14 \%$ & 2435 & 158 & $\mathrm{DC}$ & 66,29 & 9,45 & 14,60 \\
\hline SUN et a t [17] & 300 & 150 & 1800 & 600 & 258 & 25 & 340 & 45 & $119 \%$ & $131 \%$ & Barra AçO & 14 & $0,71 \%$ & 472 & 200 & EC - ELA & 0,00 & $\mathrm{NI}$ & 28,64 \\
\hline Sun etal. [1/] & 300 & 150 & 1800 & 600 & 258 & 25 & 340 & 45 & $1,19 \%$ & $1,31 \%$ & Barra CFRP & 8 & $0,23 \%$ & 2629 & 147 & ELA - DC & 78,27 & $\mathrm{NI}$ & 14,87 \\
\hline WAHAB et a [18] & 250 & 150 & 2000 & 600 & 185 & 50 & 510 & 60 & $0.57 \%$ & $105 \%$ & Barra CFRP - & 9 & $0,18 \%$ & 1970 & 136 & CE & 48,40 & $N 1$ & $\mathrm{NI}$ \\
\hline VIATABD EL DI. [10] & 200 & 1000 & 2000 & 000 & 105 & so & 510 & 00 & 每, & $1,003 \%$ & Dalid Crner & 9,5 & $0,20 \%$ & 2166 & 130 & & 44,34 & & $\mathrm{INI}$ \\
\hline & & & & & & & & & & & Barra CFRP & & $012 \%$ & 2350 & 165 & CE & 43,64 & 10,03 & 31,70 \\
\hline & & & & & & & & & & & Barra GFRP & 8 & $0,12 \%$ & 1350 & 60 & RC - DCTC - DE & 39,68 & 16,28 & 59,70 \\
\hline SHARAKY et al. [19] & 280 & 160 & 2400 & 800 & 236 & 30 & 545 & 30 & $0,60 \%$ & $0,63 \%$ & Barra CFRP & $0^{\circ}$ & $023 \%$ & 2350 & 165 & $\mathrm{DC}$ & 46,84 & 6,02 & 20,30 \\
\hline & & & & & & & & & & & Barra GFRP & & $0,25 \%$ & 1350 & 60 & DCTC & 44,88 & 16,33 & 42,40 \\
\hline & & & & & & & & & & & Barra GFRP & 12 & $0,26 \%$ & 1350 & 60 & $\mathrm{CE}$ & 42,32 & 11,87 & 35,30 \\
\hline & & & & & & & & & & & & 6 & $0,09 \%$ & 650 & & & 26,00 & & 41,68 \\
\hline HOSEN et al. [20] & 250 & 125 & 2000 & 650 & 211 & 27 & 550 & 40 & $0,86 \%$ & $0,90 \%$ & Barra Aço & 8 & $0,16 \%$ & 536 & 200 & EC - ELA & 32,83 & $\mathrm{NI}$ & 40,23 \\
\hline & & & & & & & & & & & & 10 & $0,26 \%$ & 570 & & & 36,99 & & 39,13 \\
\hline & & & & & & & & & & & & $1,2 \times 25$ & $0,08 \%$ & & & CE & 78,00 & & 9,50 \\
\hline IBRAHIM et al. [21] & 400 & 200 & 1800 & 600 & 333 & 50 & 560 & 25 & $0,24 \%$ & $1,13 \%$ & Fita CFRP & $1,2 \times 37,5$ & $0,12 \%$ & N/A & 165 & $\mathrm{CE}$ & 93,00 & $\mathrm{NI}$ & 12,00 \\
\hline & & & & & & & & & & & & $1,2 \times 50$ & $0,16 \%$ & & & $\mathrm{CE}$ & 87,00 & & 14,00 \\
\hline PENG et al. [22] & 350 & 150 & 2900 & 1200 & 309 & 25 & $\mathrm{~N} / \mathrm{A}$ & 30 & $0,87 \%$ & $0,67 \%$ & Fita CFRP & $16 \times 2$ & $0,13 \%$ & 2068 & 131 & CE & 75,72 & $\mathrm{NI}$ & 45,79 \\
\hline REZAZADEH et al. [23] & 300 & 150 & 2200 & 1050 & 264 & 25 & 585 & 32,2 & $0,40 \%$ & $0,47 \%$ & Fita CFRP & $20 \times 1,4$ & $0,06 \%$ & 1922 & 164 & $E C-R C$ & 48,81 & $\mathrm{NI}$ & 24,46 \\
\hline & & & & & & & & & & & Barra CFRP & 8 & $0,23 \%$ & 2350 & 170 & DC & 46,88 & 6,00 & 20,30 \\
\hline SHARAKY et al [24] & 280 & 160 & 2400 & 800 & 236 & 30 & 540 & 30 & $0.60 \%$ & $0.63 \%$ & Fita CFRP & $20 \times 1,4$ & $0,13 \%$ & 2500 & 165 & $\mathrm{CE}$ & 44,68 & 8,00 & 24,70 \\
\hline SHARAKY et al. [24] & 280 & 160 & 2400 & 800 & 236 & 30 & 540 & 30 & $0,60 \%$ & 0,63\% & Rarra GERP & 12 & $0,23 \%$ & 1350 & 64 & DCTC & 44,88 & 13,00 & 42,40 \\
\hline & & & & & & & & & & & Barra GFRP & 12 & $0,26 \%$ & 1330 & 64 & CE & 42,32 & 12,00 & 35,30 \\
\hline WU et a [25] & 300 & 150 & 1800 & 600 & 253 & 30 & 340 & 34.4 & $1.22 \%$ & $131 \%$ & Barra CERP & 8 & $0,12 \%$ & 2629 & 170 & ELA - EC & 77,01 & Ne & 25,60 \\
\hline & & & & & & & & 34,4 & $1,22 \%$ & $1,31 \%$ & & & $0,23 \%$ & & & CE & 78,27 & $\mathrm{NI}$ & 14,90 \\
\hline B BLOTTA et al [26] & 160 & 120 & 2100 & 925 & 104 & 45 & 540 & 21 & $126 \%$ & $0.24 \%$ & Fita CERP & $10 \times 1.4$ & $0,15 \%$ & 2052 & 171 & $\mathrm{RCl}$ & 14,94 & N1 & 30,00 \\
\hline DILUTIA EL dI. [LO] & 100 & 120 & 2100 & 923 & 104 & 45 & & 21 & $1,20 \%$ & $0,24 \%$ & Fild CFr & $10 \times 1,4$ & $0,23 \%$ & 2032 & $1 / 1$ & $\mathrm{DC}-\mathrm{RCI}$ & 15,40 & & 42,00 \\
\hline & & & & & & & & & & & Barra CERP & & $0,14 \%$ & 1588 & 1194 & $E L A-D E$ & 53,94 & & 22,00 \\
\hline & & & & & & & & & $0.43 \%$ & & Barra CFRP & & $0,27 \%$ & 1588 & 119,4 & $E L A-D E$ & 72,54 & & 26,00 \\
\hline EL-GAMAL et al . [27] & 300 & 200 & 2360 & 930 & 261 & 25 & 190 & 10 & & $050 \%$ & Barra GFRP & 10 & $0,14 \%$ & 1185 & 5234 & ELA - EC & 48,36 & NH & 61,00 \\
\hline & 300 & 200 & 2000 & 500 & 201 & 25 & 480 & 45 & & , & Dalla Urar & & $0,27 \%$ & & 02,34 & ELA- RC- DE & 63,24 & & 54,00 \\
\hline & & & & & & & & & $0.87 \%$ & & Barra CFRP & & $0,14 \%$ & 1588 & 1194 & $E L A-D E$ & 72,54 & & 28,00 \\
\hline & & & & & & & & & & & & & $0,27 \%$ & 1588 & 119,4 & $E L A-D E$ & 86,03 & & 25,00 \\
\hline
\end{tabular}

\begin{tabular}{|c|c|c|c|c|c|c|c|c|c|c|c|c|c|c|c|c|c|c|c|}
\hline \multirow{3}{*}{ Autor } & \multicolumn{10}{|c|}{ Características geométricas e propriedades mecânicas das vigas } & \multicolumn{5}{|c|}{$\begin{array}{l}\text { Características geométricas e propriedades } \\
\text { mecânicas dos reforços NSM }\end{array}$} & \multirow{3}{*}{ Modo de Ruptura } & \multirow{3}{*}{$\begin{array}{l}\mathrm{Mu}, \exp \\
(\mathrm{kN} . \mathrm{m})\end{array}$} & \multirow{3}{*}{$\varepsilon_{t, \text { exp }}(\%)$} & \multirow{3}{*}{$\begin{aligned} \Delta u, \exp \\
(\mathrm{mm})\end{aligned}$} \\
\hline & $\mathrm{h}$ & $\mathrm{b}$ & $\mathrm{L}$ & $\mathrm{a}$ & $d$ & $\mathrm{c}_{\mathrm{t}}$ & $\frac{f_{y}}{y}$ & $f_{c}$ & $\overline{\rho_{\mathrm{s}}}$ & $\rho_{s w}$ & \multirow{2}{*}{$\begin{array}{l}\text { Tipo de } \\
\text { Mat. }\end{array}$} & $\Phi_{\mathrm{f}}$ & $\rho_{f}$ & $f_{f u}$ & $E_{f}$ & & & & \\
\hline & $\mathrm{mm}$ & $\mathrm{mm}$ & $\mathrm{mm}$ & $\mathrm{mm}$ & $\mathrm{mm}$ & $\mathrm{mm}$ & $\mathrm{MPa}$ & $\mathrm{MPa}$ & $\%$ & $\%$ & & $\mathrm{~mm}$ & $\%$ & $\mathrm{MPa}$ & Gpa & & & & \\
\hline \multirow{5}{*}{ HOSEN et al. [28] } & \multirow{5}{*}{250} & \multirow{5}{*}{125} & \multirow{5}{*}{2000} & \multirow{5}{*}{650} & \multirow{5}{*}{211} & \multirow{5}{*}{27} & \multirow{5}{*}{520} & \multirow{5}{*}{40} & \multirow{5}{*}{$0,86 \%$} & \multirow{5}{*}{$0,90 \%$} & \multirow{4}{*}{ Ba rra Aço } & 8 & $0,33 \%$ & \multirow{4}{*}{570} & \multirow{4}{*}{200} & \multirow{5}{*}{ DC } & 34,53 & \multirow{5}{*}{$\mathrm{NI}$} & 11,06 \\
\hline & & & & & & & & & & & & 10 & $0,52 \%$ & & & & 38,27 & & 9,65 \\
\hline & & & & & & & & & & & & 16 & $0,67 \%$ & & & & 0,00 & & 7,84 \\
\hline & & & & & & & & & & & & \multirow{2}{*}{12} & \multirow{2}{*}{$0,75 \%$} & & & & 44,44 & & 7,14 \\
\hline & & & & & & & & & & & Barra CFRP & & & 1850 & 124 & & 46,48 & & 12,76 \\
\hline \multirow{2}{*}{ KHALIFA [29] } & \multirow{2}{*}{260} & 150 & 2200 & 950 & 200 & 35 & 1000 & 35 & ( & $105 \%$ & Eita CERP & $25 \times 12$ & $0,16 \%$ & 2800 & 165 & CE & 43,50 & NH & 24,50 \\
\hline & & 100 & 2600 & (2) & 203 & & 400 & 年 & 0,1210 & $1,05 \%$ & 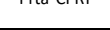 & $2 J x+2$ & $0,32 \%$ & 2000 & 105 & $\mathrm{DC}$ & 50,14 & & 17,35 \\
\hline REDA et al. [30] & 250 & 150 & 2000 & 700 & 207 & 30 & 425 & 30 & $0,51 \%$ & $0,67 \%$ & Barra GFRP & 10 & $0,44 \%$ & 940 & 55 & DCTC & 30,08 & 10,00 & 37,50 \\
\hline SHAHVERDI et al. [31] & 150 & 250 & 2000 & 800 & 113 & 25 & 508 & 55 & $0,36 \%$ & $0,27 \%$ & Fita CFRP & $20 \times 1,4$ & $0,08 \%$ & 1850 & 150 & & 9,16 & $\mathrm{NI}$ & 55,90 \\
\hline & & & & & & & & & & & & 8 & $0,33 \%$ & & & $R C-E C$ & 46,16 & & 22,27 \\
\hline SHUKRI et al. [32] & 250 & 125 & 2000 & 650 & 211 & 27 & 520 & 40 & $0,86 \%$ & $0,90 \%$ & Barra CFRP & 10 & $0,52 \%$ & 1850 & 124 & RC-EC & 57,45 & $\mathrm{NI}$ & 24,03 \\
\hline & & & & & & & & & & & & 12 & $0,75 \%$ & & & $\mathrm{DC}$ & 56,23 & & 16,89 \\
\hline & & & & & & & & & & & Barra CFRP & $5,9 \times 5,9$ & $0,06 \%$ & 1500 & 100 & $\mathrm{RC}$ & 41,53 & 15,00 & 58,68 \\
\hline & & & & & & & & & & & & $1,4 \times 15$ & $0,04 \%$ & & & $\mathrm{RC}$ & 41,16 & 14,80 & 58,94 \\
\hline JUNG et al [33] & 300 & 200 & 3000 & 1050 & 260 & 25 & 436 & 27 & $0.45 \%$ & $079 \%$ & Fita CFRP & $14 \times 25$ & $0,06 \%$ & 2482,5 & 167 & $\mathrm{CE}$ & 45,24 & 12,28 & 53,98 \\
\hline Jung et al. [33] & 300 & 200 & 3000 & 1050 & 200 & & 436 & & $0,45 \%$ & $0,19 \%$ & & $1,4 \times 25$ & $0,12 \%$ & & & $D C$ & 57,57 & 10,21 & 46,92 \\
\hline & & & & & & & & & & & Rarra CEPD & 0 & $0,11 \%$ & 1879 & 12102 & CE & 48,63 & 13,02 & 43,88 \\
\hline & & & & & & & & & & & Barra CFRP & 9 & $0,22 \%$ & $18 / 9$ & 121,42 & $D C$ & 59,04 & 10,85 & 39,50 \\
\hline SHARAKY et al. [34] & 250 & 150 & 2000 & 700 & 207 & 30 & 425 & 32,4 & $0,51 \%$ & $0,67 \%$ & Barra GFRP & 10 & $0,44 \%$ & 940 & 55 & DCTC & 30,08 & $\mathrm{NI}$ & 37,50 \\
\hline
\end{tabular}


As vigas reforçadas com CFRP apresentaram os maiores acréscimos de momento de ruptura ( $\left.\mathrm{M}_{\mathrm{u}, \exp }\right)$ em relação às vigas sem reforço, enquanto as vigas reforçadas com GFRP apresentaram os maiores incrementos de ductilidade, conforme as flechas registradas nos ensaios; - O comprimento do reforço colado interfere no modo e na carga de ruptura da viga reforçada. Vigas reforçadas com barra ou fita de CFRP romperam por destacamento do cobrimento do concreto para razão menor que $90 \%$ entre o comprimento do reforço colado e o vão livre da viga; - A resistência do concreto à compressão influência o modo de ruptura. Vigas com resistência do concreto à compressão mais elevada tenderam a ter o descolamento do reforço sob cargas de ruptura um pouco maiores; - A taxa e a configuração do reforço têm grande influência no comportamento das vigas. Vigas com maior taxa de reforço apresentam maior carga de ruptura, menor ductilidade e ruptura sem aviso prévio.

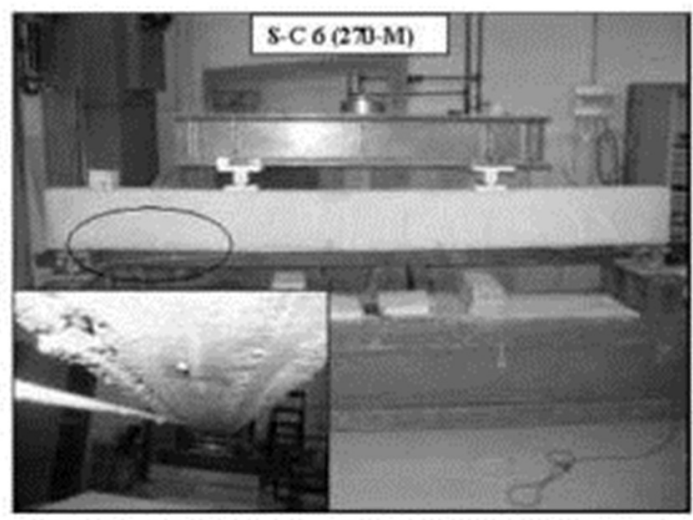

(a)

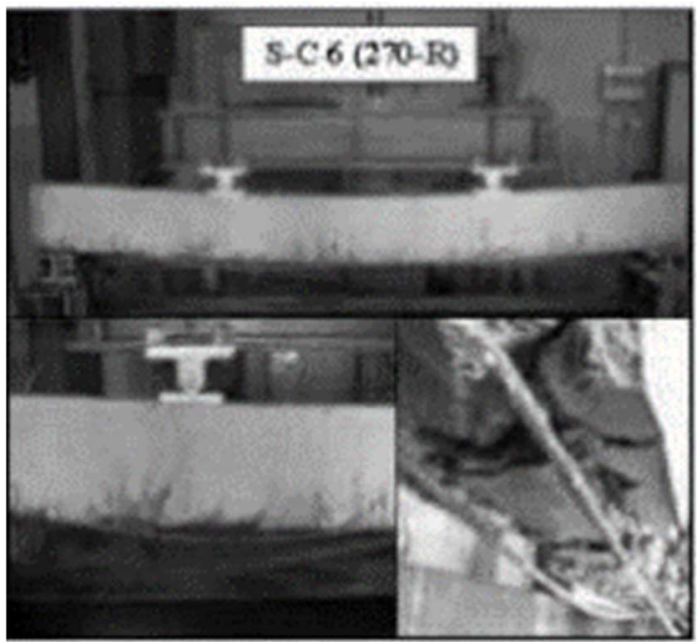

(c)

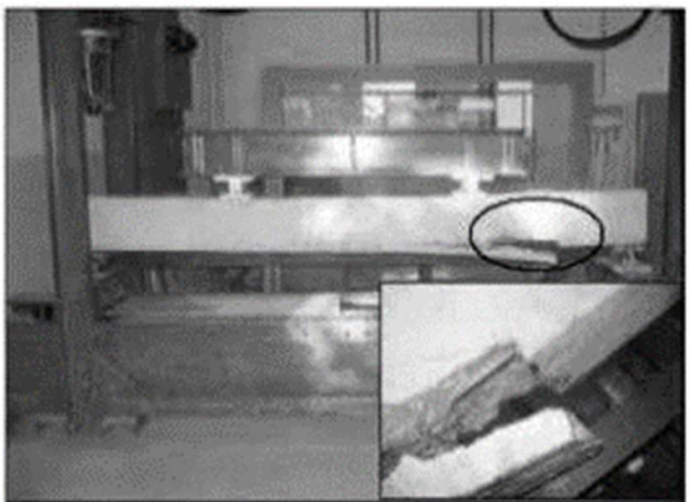

(b)

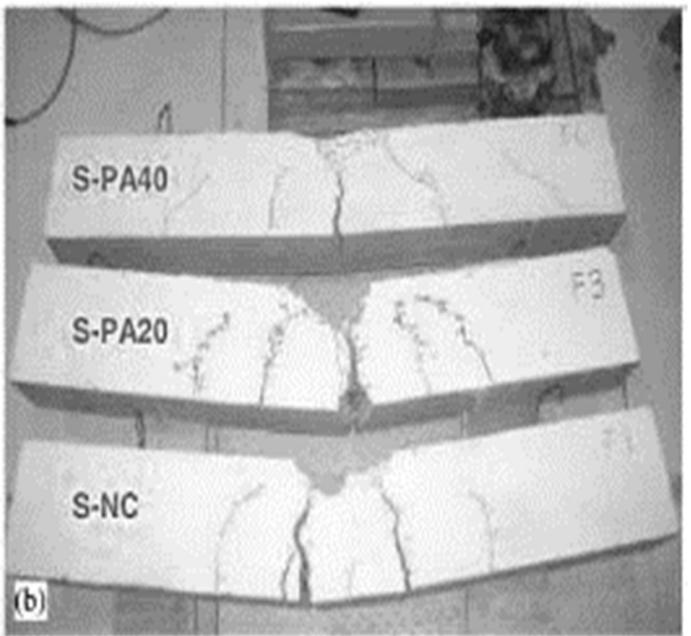

(d)

Figura 3: Modos de ruptura: (a) tipo CE, (b) tipo DC, (c) tipo DCTC e (d) tipo RC. Fonte: Al-Mahmoud et al. (2009).

\section{METODOLOGIA}

\section{Método de dimensionamento de reforço à flexão}

Conforme a ABNT NBR 6118:2014 (TANG et al., 2005), o dimensionamento de uma viga de concreto armado deve garantir segurança à estrutura em relação ao Estado Limite Último, devendo sua resistência final ser igual ou superior às suas solicitações. Sendo assim, para fins de projeto, ocorre a majoração das 
ações e a minoração da capacidade resistente dos materiais envolvidos, de acordo com o ambiente e a destinação da estrutura.

Segundo Carneiro (2004), o dimensionamento à flexão de vigas de concreto armado reforçadas com FRP deve ser feito de modo a se obter uma ruptura dúctil por flexão. Devem ser impedidas rupturas frágeis, por exemplo, as acarretadas por: ancoragem deficiente da armadura longitudinal interna de tração; por tração ou compressão do concreto da mesa, em decorrência da escassez de armadura transversal interna; por esmagamento da biela de concreto, ou por descolamento e/ou destacamento do reforço.

Na Tabela 2 são apresentadas as normas disponíveis na literatura para o dimensionamento à flexão de vigas de concreto armado reforçadas com materiais compósitos de resina e fibras. Tais normas não tratam, especificamente, do reforço do tipo NSM, sendo assim necessárias adaptações. Os estudos tomam por base hipóteses fundamentais da teoria da flexão simples, que levam em consideração a manutenção das seções planas até a ruptura; a perfeita aderência e a compatibilidade de deformações entre o concreto e o material compósito e as curvas tensão - deformação dos materiais.

Tabela 2: Normas sobre dimensionamento de reforço à flexão de vigas.

\begin{tabular}{|l|l|}
\hline \multicolumn{1}{|c|}{ Norma } & \multicolumn{1}{c|}{ Descrição } \\
\hline $\begin{array}{l}\text { ACI 440.2R-08 } \\
(2008)\end{array}$ & $\begin{array}{l}\text { Guia para o projeto e construção de sistemas de PRF ligados externamente para o fortalecimento de } \\
\text { estruturas de concreto. }\end{array}$ \\
\hline $\begin{array}{l}\text { FIB BULLETIN 14 } \\
(2001)\end{array}$ & FRP como reforço ligado externamente de estruturas R C: base do design e conceito de segurança \\
\hline $\begin{array}{l}\text { ISIS CANADA. } \\
\text { MANUAL NO. } 4 \\
(2001)\end{array}$ & $\begin{array}{l}\text { Reforço de estruturas de concreto armado com polímeros reforçados por fibras (FRPs) ligados } \\
\text { externamente. }\end{array}$ \\
\hline $\begin{array}{l}\text { JAPAN SOCIETY OF } \\
\text { CIVIL ENGINEERS } \\
(1997)\end{array}$ & $\begin{array}{l}\text { Recomendação para projeto e construção de estruturas de concreto utilizando materiais contínuos de } \\
\text { reforço de fibras. }\end{array}$ \\
\hline $\begin{array}{l}\text { CNR DT 200/2004 } \\
\text { (2004) }\end{array}$ & $\begin{array}{l}\text { Diretrizes para projeto, execução e controle de intervenções de fortalecimento por meio de materiais } \\
\text { compósitos reforçados com fibras, concreto armado e estruturas de concreto protendido, estruturas de } \\
\text { alvenaria. Conselho Nacional de Pesquisa, Comitê Consultivo em Regulamentações Técnicas para } \\
\text { Construções }\end{array}$ \\
\hline
\end{tabular}

Para o caso do reforço tipo NSM, as seguintes equações de equilíbrio foram deduzidas, considerando ruptura dúctil e a distribuição de deformações e tensões da Figura 4, conforme previsto na NBR 6118:2014 (2014):

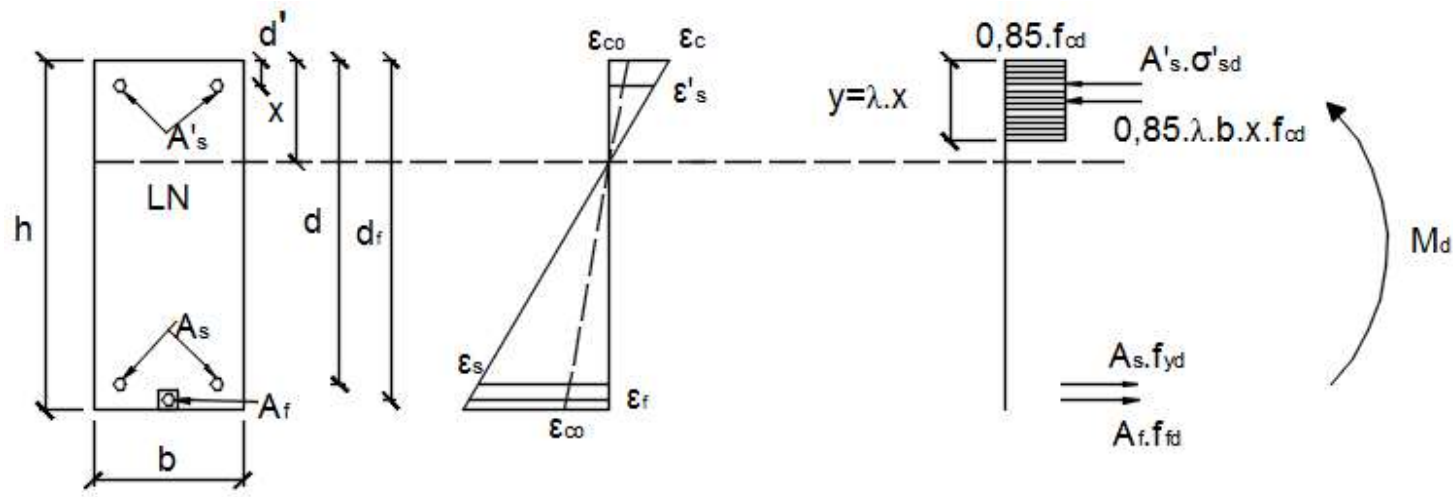

Figura 4: Distribuição de deformações e tensões na seção transversal da viga reforçada, no Estado Limite Último. 


$$
\begin{aligned}
& \sum F=0=>\alpha \cdot b \cdot y \cdot f_{c d}+A^{\prime}{ }_{s} \cdot \sigma^{\prime}{ }_{s d}=A_{s} \cdot f_{y d}+A_{f} \cdot f_{f d} \\
& f_{c d}=\frac{f_{c k}}{\gamma_{c}}, \operatorname{com} \gamma_{c}=1,4 \\
& f_{y d}=\frac{f_{y k}}{\gamma_{s}}, \operatorname{com} \gamma_{s}=1,15 \\
& f_{f d}=\frac{E_{f} \cdot \varepsilon_{f}}{\gamma_{f}} \\
& \sum M_{d}=0 \text { em } A_{s} \\
& M_{d}=\alpha \cdot b \cdot y \cdot f_{c d} \cdot(d-0,5 \cdot y)+A^{\prime}{ }_{s} \cdot \sigma^{\prime}{ }_{s d} \cdot\left(d-d^{\prime}\right)+A_{f} \cdot f_{f d} \cdot\left(d_{f}-d\right) \\
& y=\frac{\left(A_{s} \cdot f_{y d}+A_{f} \cdot f_{f}\right)}{0,85 \cdot b \cdot f_{c k}} \\
& y=x \\
& \text { para } f_{c k} \\
& \leq 50 \mathrm{MPa} \\
& =0,8-\left(f_{c k}-50\right) / 400, \text { para } f_{c k}>50 M P a\left(f_{c k} \text { em MPa }\right) \\
& \alpha=0,85 \text { para concretos com } f_{c k} \leq 50 \mathrm{MPa} \\
& \alpha=0,85 .\left(1,0-\frac{f_{c k}-50}{200}\right) \text { para } 50 M P a<f_{c k} \leq 90 M P a\left(f_{c k} \text { em MPa }\right) \\
& \sigma_{s d}^{\prime}=\varepsilon^{\prime}{ }_{s} \cdot E_{s} \leq f^{\prime}{ }_{y d} \\
& f^{\prime}{ }_{y d}=\frac{f^{\prime}{ }_{y k}}{\gamma_{s}}, \operatorname{com} \gamma_{s}=1,15 \text {; } \\
& \varepsilon^{\prime}{ }_{s u}=\frac{x_{u}-d^{\prime}}{x_{u}} \cdot \varepsilon_{c u}
\end{aligned}
$$

Onde:

Md é o momento de cálculo resistente;

b, d, xe h são a largura, a altura útil, a altura da linha neutra na ruptura e a altura total da viga, respectivamente; $\varepsilon_{c}$ e $f_{c d}$ são a deformação específica e a resistência à compressão de projeto do concreto, respectivamente; $\varepsilon_{o}$ é a deformação do bordo mais tracionado da viga antes da execução do reforço; $A_{s}$ e $f_{y d}$ são a área da seção transversal e a resistência ao escoamento de projeto da armadura longitudinal interna de tração, respectivamente;

$A_{s}^{\prime}, \sigma_{s d}^{\prime}, f_{y d}^{\prime}, \varepsilon_{s}^{\prime}$ e $E_{s}$ são a área da seção transversal, a tensão de projeto, a resistência ao escoamento de projeto, a deformação específica e o módulo de elasticidade da armadura longitudinal interna de compressão, respectivamente; $\mathrm{A}_{f}, \mathrm{f}_{\mathrm{fd}}, \mathrm{E}_{\mathrm{f}}$ e $\varepsilon_{f}$ são a área da seção transversal, a resistência à tração de projeto, o módulo de elasticidade e a deformação específica de projeto do material de reforço, respectivamente.

Para a análise da influência da taxa $\left(\rho_{f}\right)$ e do tipo de reforço na deformação específica do reforço, elaboraram se os gráficos da Figura 5 a partir dos resultados das pesquisas apresentadas na Tabela 1 para barras de CFRP e GFRP. Verifica-se que a deformação do reforço na viga $\left(\varepsilon_{f}\right)$ diminui em relação à deformação última do mesmo $\left(\varepsilon_{\mathrm{fu}}\right)$, com o aumento da taxa de reforço. A deformação última do reforço foi obtida por meio de ensaios de tração axial do material. Além disso, para uma mesma taxa de reforço, a relação $\varepsilon_{f} / \varepsilon_{\mathrm{fu}}$ é superior nos reforços de GFRP em relação ao de CFRP, acarretando maiores deformações do material. 


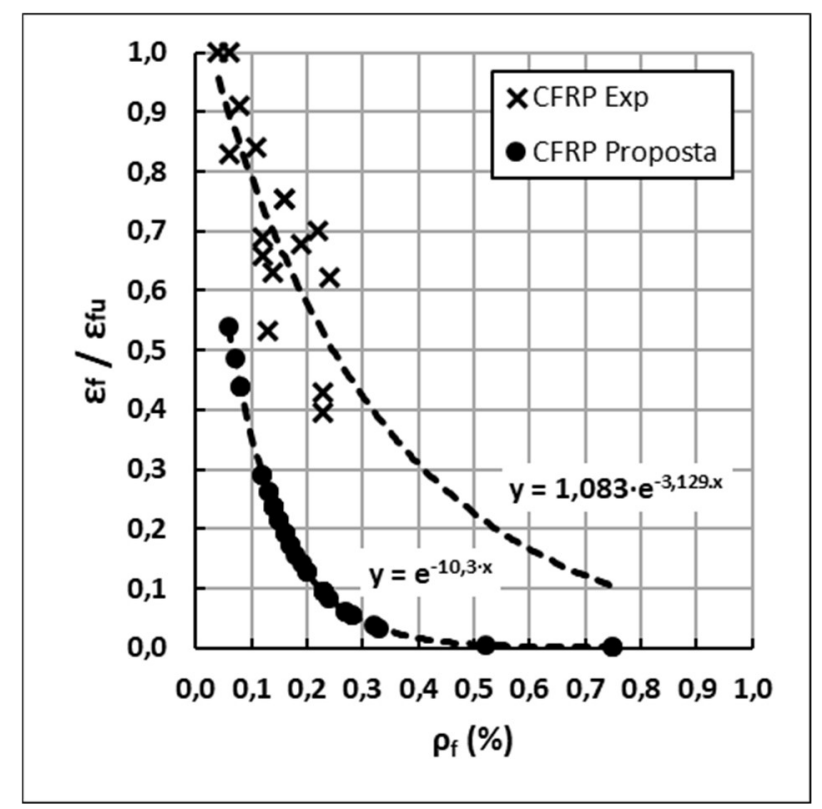

Figura 5: Gráfico de $\varepsilon_{\mathrm{f}} / \varepsilon_{\mathrm{fu}}$ experimental e versus taxa de reforço de CFRP e GFRP.

Carneiro (2004) propõe que a deformação última do reforço $\left(\varepsilon_{f}\right)$ seja limitada a 0,005 para evitar rupturas prematuras das vigas por destacamento do concreto no bordo reforçado. Nos trabalhos experimentais, as deformações do reforço na ruptura das vigas ultrapassaram o limite de $5 \%$, sendo assim, foram propostas novas curvas, limitando-se os valores de deformação do reforço com a finalidade de evitar as rupturas prematuras das vigas por destacamento do concreto. As curvas propostas são representadas na Figura 6 e na Figura 7. Nas curvas correspondentes a CFRP Exp e GFRP Exp considerou-se os valores de $\varepsilon_{f}$ obtidos experimentalmente e nas curvas CFRP Proposta e GFRP Proposta limitou-se os valores de $\varepsilon_{\mathrm{f}}$ em função de $\rho_{\mathrm{f}}$, sendo sempre menor do que $5 \%$.

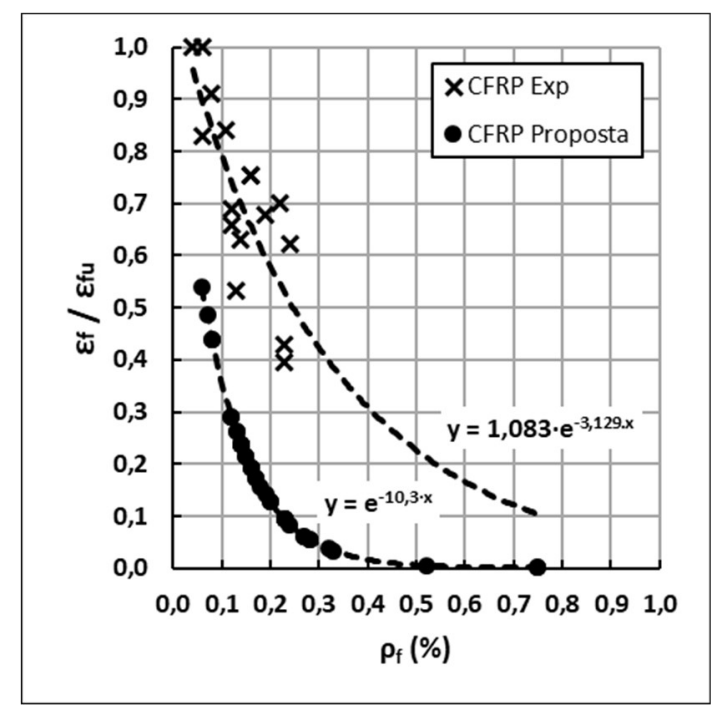

Figura 6: Gráfico de $\varepsilon_{\mathrm{f}} / \varepsilon_{\mathrm{fu}}$, experimental e limite, versus taxa de reforço de CFRP.

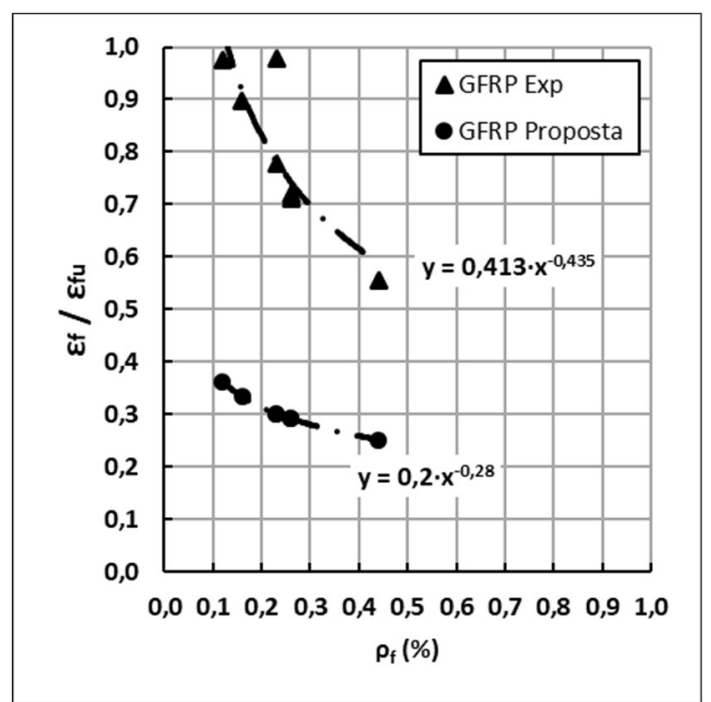

Figura 7: Gráfico de $\varepsilon_{\mathrm{f}} / \varepsilon_{\mathrm{fu}}$ experimental e limite versus taxa de reforço de GFRP.

Os pesquisadores não apresentaram as deformações do reforço de aço na ruptura das vigas, porém comparando-se o $M_{u, \text { exp }}$ com $M_{u, c a l c}$ da viga, onde $M_{u, \text { exp }}$ é o momento último resistente da viga obtida experimentalmente e $\mathrm{M}_{\mathrm{u}, \text { calc }}$ é calculado considerando $\mathrm{f}_{\mathrm{f}}$ igual à tensão de escoamento do aço, nota-se que 
os resultados ficaram muito próximos. A Figura 8 mostra de forma gráfica a boa aproximação entre os resultados $\mathrm{M}_{\mathrm{u}, \mathrm{exp}}$ e $\mathrm{M}_{\mathrm{u}, \mathrm{calc}}$.

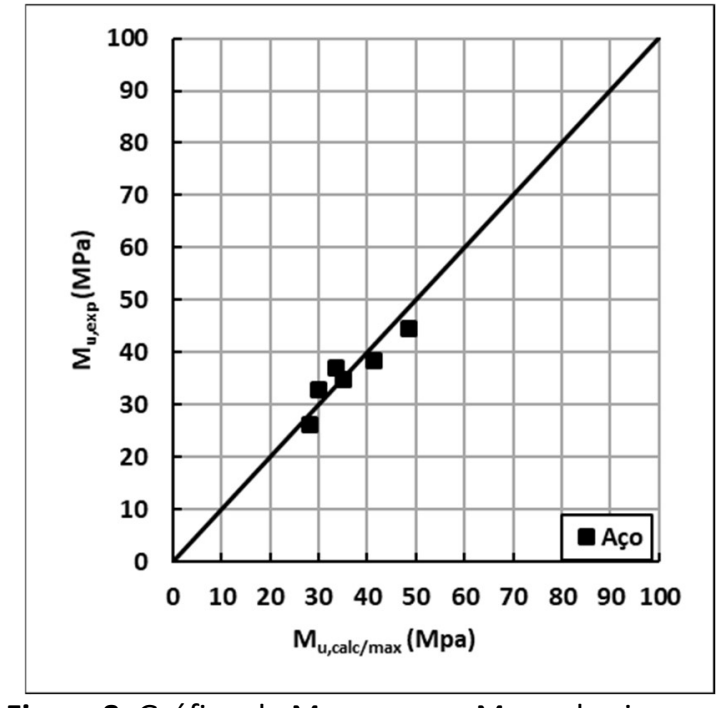

Figura 8: Gráfico de $\mathrm{M}_{\mathrm{u}, \mathrm{exp}}$ versus $\mathrm{M}_{\mathrm{u}, \mathrm{calc}}$ da viga com reforço em aço.

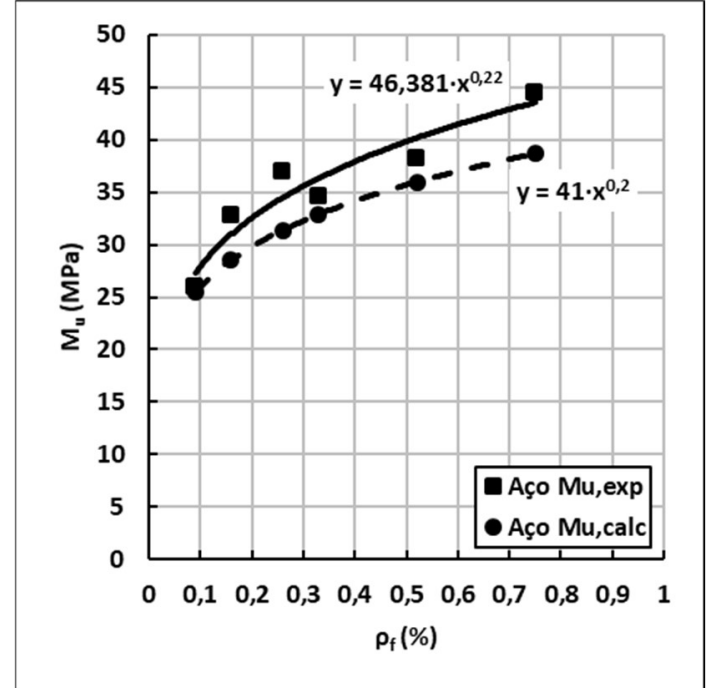

Figura 9: Gráfico de $M_{u}$ versus taxa de reforço em aço.

Com a finalidade de propor uma expressão para o momento calculado em função da taxa de reforço em barras de aço da viga, foi traçado o gráfico dos $M_{u, \text { exp }}$ e $M_{u, c a l c}$ em função do taxa de reforço conforme Figura 9. O $\mathrm{M}_{\mathrm{u}, \text { calc }}$ foi obtido limitando a tensão de ruptura do reforço à tensão de escoamento do aço. A diferença percentual entre $M_{u, e x p}$ e $M_{u, c a l c}$ aumentou com o aumento da taxa de reforço. Essa diferença foi de $8,03 \%$, para $\rho_{f}=0,1$ e $12,47 \%$, para $\rho_{f}=0,75$.

\section{Avaliação de flecha imediata}

O item 3.2.4 da NBR 6118:2014 (2014) trata sobre o estado limite das deformações excessivas, calculadas conforme o item 17.3.2. Os limites estipulados para a utilização normal da estrutura são dados no item 13.3. As principais propriedades dos materiais que influenciam na deformação da estrutura carregada são o módulo de elasticidade e a resistência à tração dos materiais empregados. Dependendo da intensidade do carregamento, as seções transversais podem se encontrar no estádio I ou no estádio II. No primeiro estádio, o diagrama de tensões no concreto é linear em relação à distância da linha neutra. No segundo estádio, o concreto tracionado já está fissurado e as relações entre ações e deslocamentos não são lineares.

No estádio II, para vigas não reforçadas, considera-se que os esforços de tração são resistidos apenas pela armadura posicionada na região tracionada, sendo o concreto fissurado desprezado. Para o cálculo do momento de inércia, é necessário conhecer a posição da $L N,\left(x_{I I}\right)$, obtida igualando-se o momento estático da seção homogênea a zero. Nas vigas com seção retangular reforçadas à flexão pelo método NSM, propõese a verificação prévia da flecha incorporando o material de reforço nas equações propostas pela ABNT NBR 6118:2014 (2014). Sendo assim, as tensões e deformações na seção retangular da viga no estádio II são mostradas na Figura 10. 

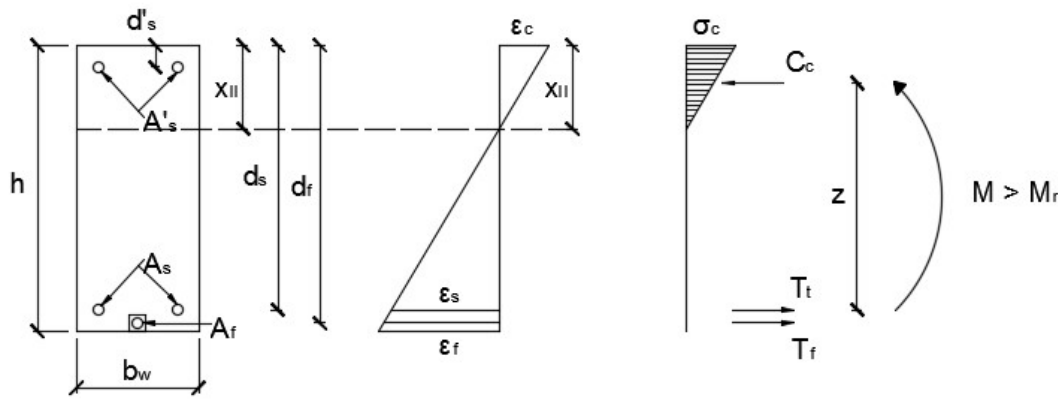

Figura 10: Distribuição de deformações e tensões na seção transversal da viga reforçada, no Estádio II.

$$
\begin{aligned}
& b_{w} / 2 \cdot x_{I I}^{2}+\left(\left(\alpha_{e}-1\right) \cdot A_{s}^{\prime}+\alpha_{e} \cdot A_{s}+\alpha_{e, f} \cdot A_{f}\right) \cdot x_{I I}-\left(d_{s}^{\prime} \cdot\left(\alpha_{e}-1\right) \cdot A_{s}^{\prime}+d_{s} \cdot \alpha_{e} \cdot A_{s}+d_{f} \cdot \alpha_{e, f} \cdot A_{f}\right)=0 \\
& x_{I I}=\frac{-\left(\left(\alpha_{e}-1\right) \cdot A_{s}^{\prime}+\alpha_{e} \cdot A_{s}+\alpha_{e, f} \cdot A_{f}\right)}{b_{w}}+\frac{\sqrt{\left(\left(\alpha_{e}-1\right) \cdot A_{s}^{\prime}+\alpha_{e} \cdot A_{s}+\alpha_{e, f} \cdot A_{f}\right)^{2}+2 \cdot b_{w} \cdot\left(d_{s}^{\prime} \cdot\left(\alpha_{e}-1\right) \cdot A_{s}^{\prime}+d_{s} \cdot \alpha_{e} \cdot A_{s}+d_{f} \cdot \alpha_{e, f} \cdot A_{f}\right)}}{b_{w}} \\
& I_{I I}=b_{w} \cdot x_{I I}^{3} / 3+\alpha_{e} \cdot A_{s} \cdot\left(d_{s}-x_{I I}\right)^{2}+\left(\alpha_{e}-1\right) \cdot A^{\prime}{ }_{s} \cdot\left(x_{I I}-d^{\prime}{ }_{s}\right)^{2}+\alpha_{e, f} \cdot A_{f} \cdot\left(d_{f}-x_{I I}\right)^{2}
\end{aligned}
$$

O momento de inércia da seção reforçada no estádio II é apresentado na equação [13]. A nova posição da $L N,\left(x_{\|}\right)$da viga reforçada de seção retangular é obtida da equação [11], sendo $x_{\|}$apresentado na equação [12]. Conforme Said (2004), o momento de inércia equivalente no estádio III, da viga totalmente fissurada, é mostrado na equação [14].

$$
I_{e}=\frac{I_{I I}}{\left[1-\left(\frac{I_{I I}}{I_{e, y}}\right) \cdot\right]}
$$

onde:

$$
\begin{aligned}
& I_{e, y}=\frac{I_{I I}}{\left[1-\left(1-\frac{I_{I I}}{I_{c}}\right) \cdot\left(\frac{M_{c r}}{M_{y}}\right)^{2}\right]} ; \\
& \left\{\begin{array}{l}
\left(\frac{M_{y}}{M_{I I I}}\right)^{2}, \text { para carregamento a três pontos ou quatro pontos; } \\
\left(\frac{M_{y}}{M_{I I I}}\right) \cdot\left(1-\sqrt{1-\frac{M_{y}}{M_{I I I}}}\right), \text { para carregamento uniforme; }
\end{array}\right.
\end{aligned}
$$

$\alpha_{e}=E_{s} / E_{c}$ e $\alpha_{e, f}=E_{f} / E_{c}$;

$\mathrm{I}_{c}$ é o momento de inércia da seção bruta de concreto;

II é o momento de inércia da seção fissurada de concreto no estádio II;

$\mathrm{M}_{\mathrm{cr}}$ é momento fletor máximo de fissuração do elemento estrutural;

$M_{y}$ é o momento fletor máximo no escoamento da armadura interna do aço, estimado em 0,6. $\mathrm{M}_{\mathrm{d}}$;

M III é o momento fletor no estádio III;

$\mathrm{E}_{\mathrm{cs}}$ é o módulo de elasticidade secante do concreto.

Assim, a flecha imediata ou instantânea (sem efeito da fluência) no meio do vão, para vigas com duas cargas concentradas equidistantes entre os apoios, conforme Said (2004), pode ser calculada a partir da seguinte equação [15].

$$
\Delta=\frac{(P / 2) \cdot a}{24 \cdot E_{C} \cdot I_{e}} \cdot\left(3 \cdot L^{2}-4 \cdot a^{2}\right)
$$

onde:

P é a carga total aplicada, definida para certa combinação de ações;

a é o vão de cisalhamento da viga;

L é o vão livre da viga. 


\section{RESULTADOS E DISCUSSÃO}

Valores teóricos de momento fletor último, momento fletor de fissuração e flecha última das vigas pesquisadas

O momento fletor último e o momento de fissuração no meio do vão das vigas reforçadas foram calculados conforme a metodologia prevista nos itens 3.1 e 3.2 e adotando os coeficientes de majoração igual a 1. A flecha última foi calculada com a Eq [15] a partir da carga P obtida a partir do momento fletor último e do momento de inércia da viga no estádio III. A Tabela 3 apresenta os resultados obtidos. Verificase que o momento e a flecha experimental apresentaram-se em média 159\% e $260 \%$ superiores ao momento e à flecha teóricos, respectivamente, demonstrando que as equações propostas são favoráveis à segurança das peças.

Tabela 3: Resultados teórico das vigas das pesquisas (Continua).

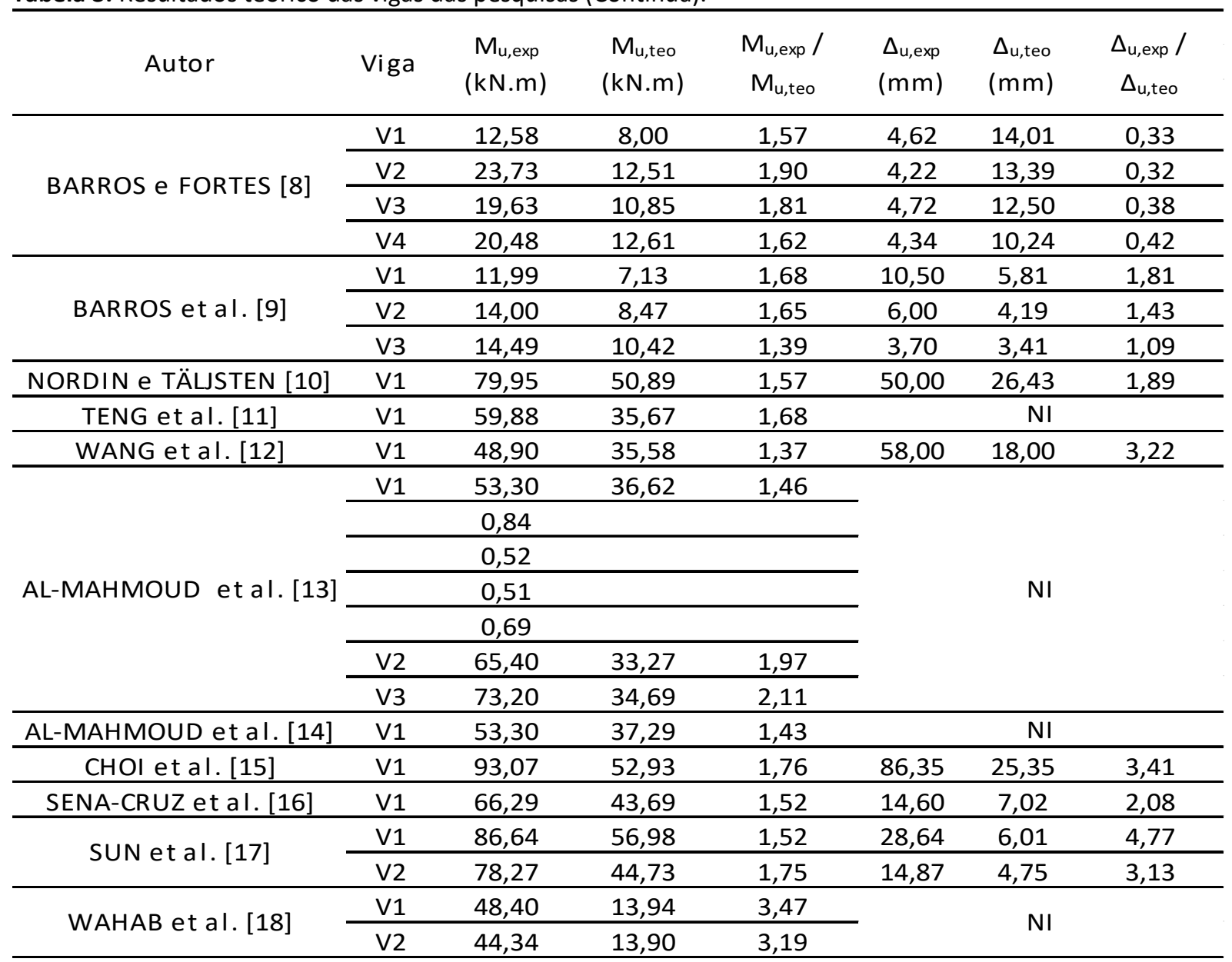

Tabela 3: Resultados teórico das vigas das pesquisas (Continuação). 


\begin{tabular}{|c|c|c|c|c|c|c|c|}
\hline Autor & Viga & $\begin{array}{c}M_{u, \exp } \\
(k N . m)\end{array}$ & $\begin{array}{c}\mathrm{M}_{\mathrm{u}, \mathrm{teo}} \\
(\mathrm{kN} . \mathrm{m})\end{array}$ & $\begin{array}{c}M_{u, \exp } / \\
M_{u, \text { teo }}\end{array}$ & $\begin{array}{l}\Delta_{u, \exp } \\
(\mathrm{mm})\end{array}$ & $\begin{array}{l}\Delta_{\mathrm{u}, \text { teo }} \\
(\mathrm{mm})\end{array}$ & $\begin{array}{c}\Delta_{\mathrm{u}, \exp } / \\
\Delta_{\mathrm{u}, \text { teo }}\end{array}$ \\
\hline \multirow{5}{*}{ SHARAKY et al. [19] } & V1 & 43,64 & 35,48 & 1,23 & 31,70 & 12,31 & 2,58 \\
\hline & V2 & 39,68 & 32,01 & 1,24 & 59,70 & 11,37 & 5,25 \\
\hline & V3 & 46,84 & 32,46 & 1,44 & 20,30 & 12,40 & 1,64 \\
\hline & V4 & 44,88 & 36,85 & 1,22 & 42,40 & 13,25 & 3,20 \\
\hline & V5 & 42,32 & 37,84 & 1,12 & 35,30 & 12,27 & 2,88 \\
\hline \multirow{3}{*}{ HOSEN et al. [20] } & $\mathrm{V} 1$ & 26,00 & 26,41 & 0,98 & 41,68 & 6,85 & 6,08 \\
\hline & $\mathrm{V} 2$ & 32,83 & 27,30 & 1,20 & 40,23 & 8,04 & 5,01 \\
\hline & V3 & 36,99 & 29,14 & 1,27 & 39,13 & 8,26 & 4,74 \\
\hline \multirow{3}{*}{ IBRAHIM et al. [21] } & V1 & 78,00 & 50,02 & 1,56 & 9,50 & 6,86 & 1,39 \\
\hline & $\mathrm{V} 2$ & 93,00 & 49,35 & 1,88 & 12,00 & 7,43 & 1,62 \\
\hline & V3 & 87,00 & 46,95 & 1,85 & 14,00 & 6,26 & 2,24 \\
\hline PENG et al. [22] & V1 & 75,72 & 67,12 & 1,13 & 45,79 & 10,96 & 4,18 \\
\hline REZAZADEH et al. [23] & V1 & 48,81 & 30,66 & 1,59 & 24,46 & 10,50 & 2,33 \\
\hline \multirow{4}{*}{ SHARAKY et al. [24] } & $\mathrm{V} 1$ & 46,88 & 32,24 & 1,45 & 20,30 & 11,57 & 1,75 \\
\hline & $\mathrm{V} 2$ & 44,68 & 35,48 & 1,26 & 24,70 & 24,68 & 1,00 \\
\hline & V3 & 44,88 & 36,63 & 1,23 & 42,40 & 13,51 & 3,14 \\
\hline & V4 & 42,32 & 37,62 & 1,12 & 35,30 & 12,56 & 2,81 \\
\hline \multirow{2}{*}{ WU et al. [25] } & V1 & 77,01 & 46,95 & 1,64 & 25,60 & 6,60 & 3,88 \\
\hline & $\mathrm{V} 2$ & 78,27 & 43,06 & 1,82 & 14,90 & 6,19 & 2,41 \\
\hline \multirow{2}{*}{ BILOTTA et al. [26] } & V1 & 14,94 & 10,57 & 1,41 & 30,00 & 24,86 & 1,21 \\
\hline & $\mathrm{V} 2$ & 15,40 & 10,06 & 1,53 & 42,00 & 22,98 & 1,83 \\
\hline \multirow{6}{*}{ EL-GAMAL et al. [27] } & V1 & 53,94 & 36,03 & 1,50 & 22,00 & 9,67 & 2,27 \\
\hline & $\mathrm{V} 2$ & 72,54 & 31,78 & 2,28 & 26,00 & 11,37 & 2,29 \\
\hline & V3 & 48,36 & 33,26 & 1,45 & 61,00 & 8,29 & 7,36 \\
\hline & V4 & 63,24 & 38,81 & 1,63 & 54,00 & 9,08 & 5,95 \\
\hline & V5 & 72,54 & 61,62 & 1,18 & 28,00 & 9,50 & 2,95 \\
\hline & V6 & 86,03 & 57,59 & 1,49 & 25,00 & 11,77 & 2,12 \\
\hline \multirow{5}{*}{ HOSEN et al. [28] } & V1 & 34,53 & 29,29 & 1,18 & 11,06 & 7,29 & 1,52 \\
\hline & $\mathrm{V} 2$ & 38,27 & 32,57 & 1,17 & 9,65 & 6,97 & 1,38 \\
\hline & V3 & 35,61 & 34,86 & 1,02 & 7,84 & 5,99 & 1,31 \\
\hline & V4 & 44,44 & 36,45 & 1,22 & 7,14 & 7,03 & 1,01 \\
\hline & V5 & 46,48 & 23,23 & 2,00 & 12,76 & 9,11 & 1,40 \\
\hline \multirow{2}{*}{ KHALIFA [29] } & $\mathrm{V} 1$ & 43,50 & 26,41 & 1,65 & 24,50 & 11,62 & 2,11 \\
\hline & $\mathrm{V} 2$ & 50,14 & 21,62 & 2,32 & 17,35 & 11,24 & 1,54 \\
\hline REDA et al. [30] & V1 & 30,08 & 22,14 & 1,36 & 37,50 & 9,55 & 3,93 \\
\hline SHAHVERDI et al. [31] & V1 & 9,16 & 8,62 & 1,06 & 55,90 & 10,84 & 5,16 \\
\hline \multirow{3}{*}{ SHUKRI et al. [32] } & V1 & 46,16 & 24,54 & 1,88 & 22,27 & 11,35 & 1,96 \\
\hline & $\mathrm{V} 2$ & 57,45 & 23,49 & 2,45 & 24,03 & 12,67 & 1,90 \\
\hline & V3 & 56,23 & 23,23 & 2,42 & 16,89 & 11,05 & 1,53 \\
\hline \multirow{6}{*}{ JUNG et al. [33] } & V1 & 41,53 & 32,90 & 1,26 & 58,68 & 14,86 & 3,95 \\
\hline & $\mathrm{V} 2$ & 41,16 & 35,11 & 1,17 & 58,94 & 14,66 & 4,02 \\
\hline & V3 & 45,24 & 37,58 & 1,20 & 53,98 & 15,59 & 3,46 \\
\hline & V4 & 57,57 & 38,38 & 1,50 & 46,92 & 18,18 & 2,58 \\
\hline & V5 & 48,63 & 35,56 & 1,37 & 43,88 & 16,28 & 2,70 \\
\hline & V6 & 59,04 & 31,99 & 1,85 & 39,50 & 17,59 & 2,25 \\
\hline SHARAKY et al. [34] & V1 & 30,08 & 21,45 & 1,40 & 37,50 & 9,46 & 3,97 \\
\hline
\end{tabular}




\section{Comparação entre os resultados experimentais e teóricos}

Os gráficos de $M_{u}$ experimental em função do $M_{u}$,teórico apresentados nas Figura 11, Figura 12 e Figura 13, para material do reforço de CFRP, GFRP ou aço, respectivamente. Observou-se que ocorreu uma tendência dos pontos posicionaram-se no centro ou superior à curva média. Isso demonstra que o cálculo teórico é válido e apresenta resultados a favor da segurança. A média da razão de $M_{u, \text { exp }} / M_{u \text {,teo }}$ para os materiais analisados foram: 1,37; 1,30 e 1,20 para CFRP, GFRP e aço, respectivamente.

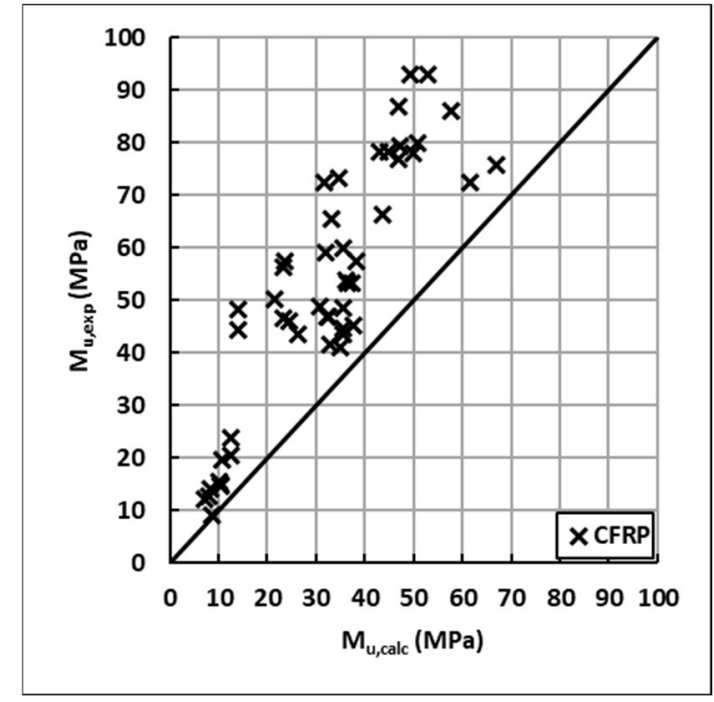

Figura 11: Gráfico Mu,exp versus $\mathrm{M}_{\mathrm{u}, \text { teo }}$, para reforço de CFRP.

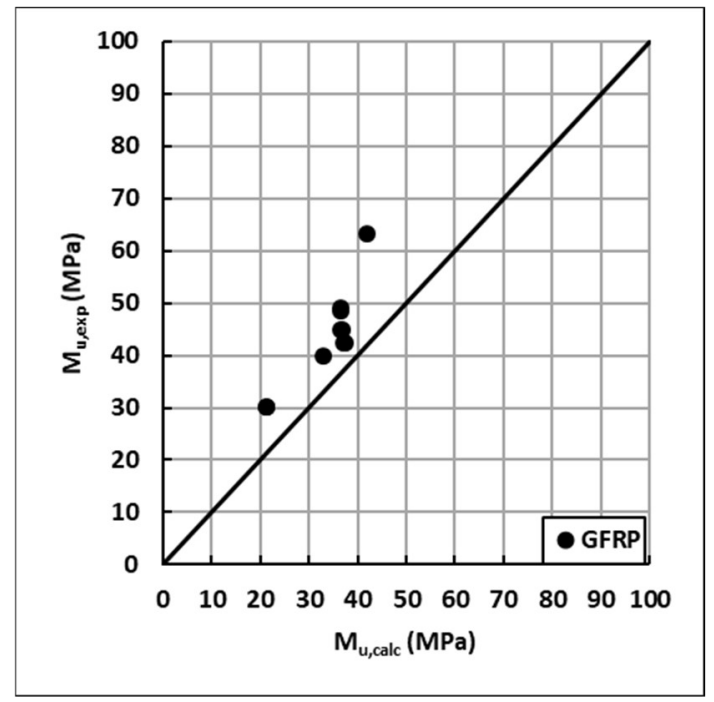

Figura 12: Gráfico $\mathrm{M}_{\mathrm{u} \text { exp }}$ versus $\mathrm{Mu}_{\mathrm{u} \text {,eo }}$, para reforço de GFRP.

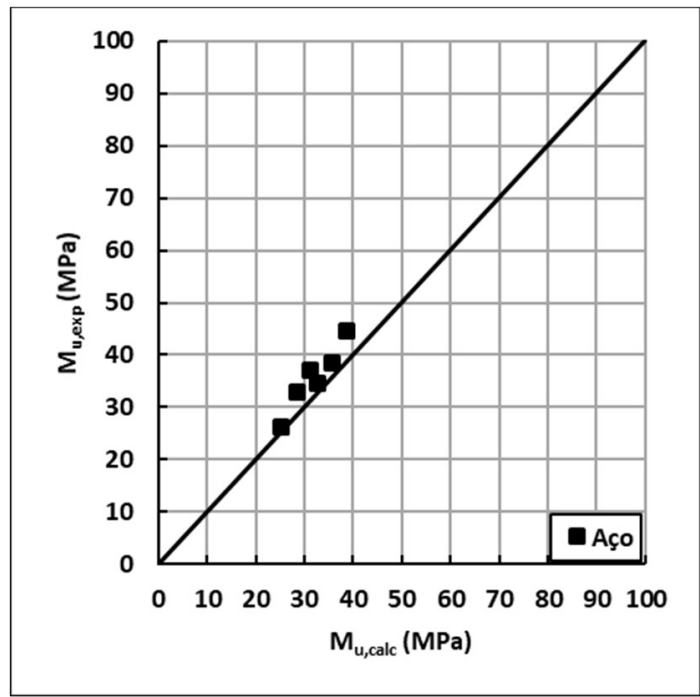

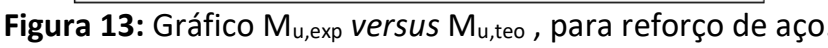

Para comparação das flechas no meio do vão, foram traçados dos gráficos de $\Delta_{u}$ experimental versus $\Delta_{u}$ teórica, os quais são apresentados na Figura 14, Figura 15 e Figura 16, respectivamente para CFRP, GFRP e aço. Também se observou uma tendência dos resultados se posicionarem superior à curva média, sendo que os reforços CFRP obtiveram diferenças menores que os materiais GFRP e aço. Os resultados médios verificados foram 1,10; 2,00 e 1,88 para os reforços CFRP, GFRP e aço, respectivamente. Em vista disso, o 
modelo teórico proposto demonstra validade e uma tendência também a favor segurança, pois o três materiais analisados obtiveram uma razão de flecha $\Delta_{\mathrm{u}, \mathrm{exp}} / \Delta_{\mathrm{u}, \text { teo }}$ acima de 1.

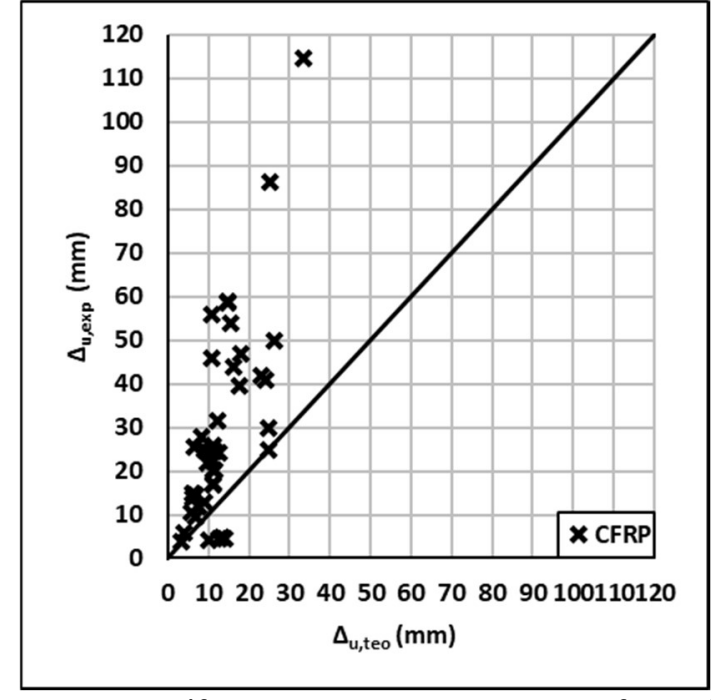

Figura 14: Gráfico $\Delta_{\mathrm{u}, \exp }$ versus $\Delta_{\mathrm{u}, \text { teo, }}$ para reforço de CFRP.

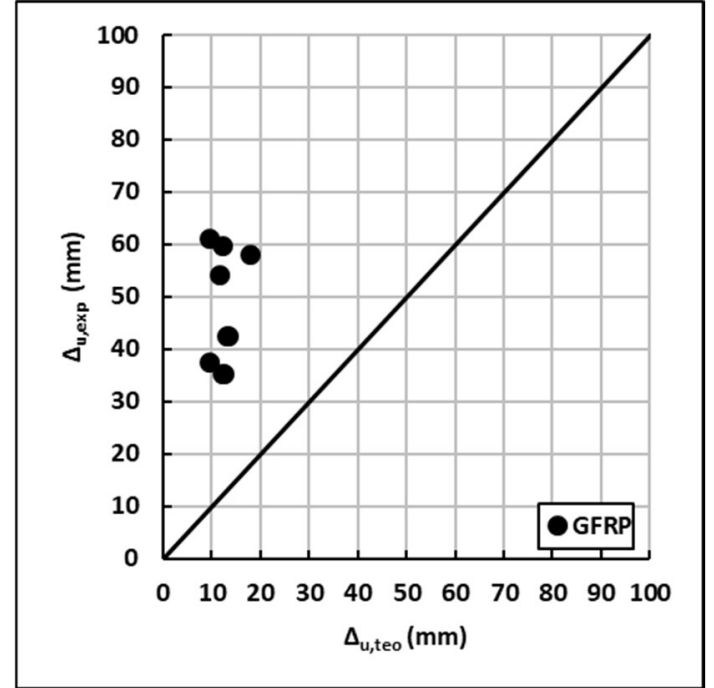

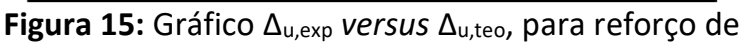
GFRP.

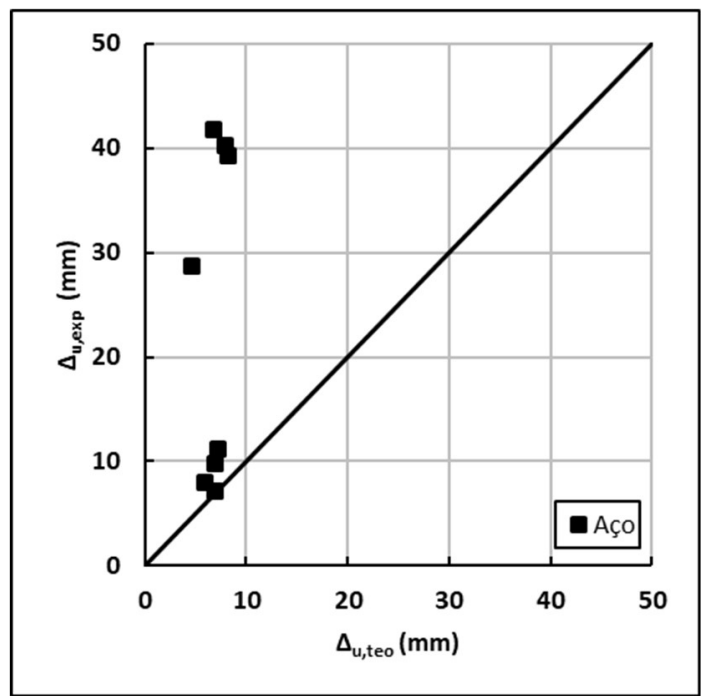

Figura 16: Gráfico $\Delta_{u, e x p}$ versus $\Delta_{u, t e o}$ para reforço de aço.

\section{CONCLUSÕES}

Os modelos teóricos utilizados para prever os momentos fletores últimos e flechas máximas à flexão em vigas com seção retangular, reforçadas com FRP ou aço pelo método NSM foram baseados em teorias da flexão simples, considerando as seções planas e a perfeita aderência entre os materiais. O modelo teórico para a previsão dos momentos últimos na ruptura, apresentaram resultados a favor a segurança com os três

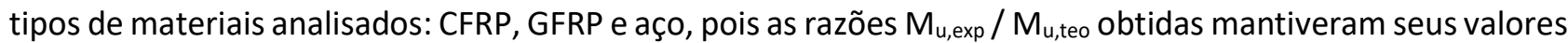
acima de 1. A deformação máxima adotada para o reforço, $\varepsilon_{f}=0,005$, demonstrou eficácia, podendo assim ser utilizada como fator usual para o limite de deformação em vigas reforçadas à flexão por meio da técnica NSM. 
Em relação ao modelo teórico para o cálculo da flecha limite no meio do vão, os resultados também foram satisfatórios, pois os resultados das razões $\Delta_{\mathrm{u}, \mathrm{exp}} / \Delta_{\mathrm{u}, \text { teo }}$ apontaram valores médios acima de 1 para os três tipos de material de reforço utilizados. Observa-se a necessidade de mais análises para os momentos fletores e flechas obtidas para a primeira fissura. Sendo assim, pode-se utilizar a teoria da flexão simples para previsão dos momentos fletores e flechas no meio do vão na ruptura em vigas retangulares reforçadas à flexão pela técnica NSM.

\section{REFERÊNCIAS}

ABNT. Associação Brasileira de Normas Técnicas. NBR 6118: Projeto de estruturas de concreto - Procedimento. Rio de Janeiro, 2014.

$\mathrm{ACl}$ 440.2R-08. Guide For The Design And Construction of Externally Bonded FRP Systems For Strengthening Concrete Structures. ACl Committee 440, Farmington Hills, 2008.

AL-MAHMOUD, F.; CASTEL, A.; FRANÇOIS, R.; TOURNEUR, C.. RC beams strengthened with NSM CFRP rods and modeling of peeling-off failure. Composite Structures, Villers-lès-Nancy Cedex, v.92, p.1920-1930, 2010.

AL-MAHMOUD, F.; CASTEL, A.; FRANÇOIS, R.; TOURNEUR, $C$.. Strengthening of RC members with near-surface mounted CFRP rods. Composite Structures, Villers-lès-Nancy Cedex, v.91, p.138-147, 2009.

BARROS, J. A. O.; DIAS, S. J. E.; LIMA, J. L. T.. Efficacy of CFRPbased techniques for the flexural and shear strengthening of concrete beams. Cement \& Concrete Composites, Guimarães, v.29, p.203-217, 2006.

BARROS, J. A. O.; FORTES, A. S.. Flexural strengthening of concrete beams with CFRP laminates bonded into slits. Cement \& Concrete Composites, Guimarães, v.27, p.471480, 2004.

BEBER, A. J.; CAMPOS FILHO, A.; CAMPAGNOLO, J. L.. Reforço de estruturas de concreto armado com tecidos préimpregnados de fibras de carbono. Revista Téchne, São Paulo, v.45, p.52-55, 2000.

BILOTTA, A.; CERONI, F.; NIGRO, E.; PECCE, M.. Efficiency of CFRP NSM strips and EBR plates for flexural strengthening of $\mathrm{RC}$ beams and loading pattern influence. Composite Structures, Napoli, v.124, p.163-175, 2015.

CARNEIRO, L. A. V.. Reforço de Vigas e Pilares de Concreto. Tese (Doutorado) - Universidade Federal do Rio de Janeiro, Rio de Janeiro, 2004.

CHOI, H. T.; WEST, J.; KHALED , S.. Partially bonded nearsurface-mounted CFRP bars for strengthened concrete Tbeams. Construction and Building Materials, Ontario, v.25, p.2441-2449, 2010.

CNR DT 200/2004. Guidelines for design, execution and control of strengthening interventions by means of fibrereinforced composites: materials, reinforced concrete and prestressed concrete structures, masonry structures. Rome: National Research Council, Advisory Committee on Technical Regulations for Constructions, 2004.
EL-GAMAL, S. E.; AL-NUAIMI, A.; AL-SAIDY, A.; AL-LAWATI, A.. Efficiency of near surface mounted technique using fiber reinforced polymers for the flexural strengthening of RC beams. Construction and Building Materials, Oman, v.118, p.52-62, 2016.

FIB BULLETIN 14. FRP as externally bonded reinforcement of R.C. structures: basis of design and safety concept. TG9.3, 2001.

HOSEN, M. D. A.; JUMAAT, M. Z.; DARAIN, K. H. M. U. D.; OBAYDULLAH, M.; ISLAM, A. B. M. S.. Flexural Strengthening of RC Beams with NSM Steel Bars. In: INTERNATIONAL CONFERENCE ON FOOD, AGRICULTURE AND BIOLOGY. Anais. Kuala Lumpur: 2014. p.8-13.

HOSEN, M. D. A.; JUMAAT, M. Z.; ALENGARAM, U. J.; ISLAM, A. B. M. S.; HASHIM, H. B.. Near Surface Mounted Composites for Flexural Strengthening of Reinforced Concrete Beams. Polymers, Kuala Lumpur, v.67, p.1-18, 2016.

IBRAHIM, W.; FATTAH, W. A.; KOTB, A.; MJEED, M. A.. Flexural behavior of rc beams strengthened with cfrp strips. In: INTERNATIONAL CONFERENCE ON FRP COMPOSITES IN CIVIL ENGINEERING. Anais. Vancouver, 2014. p.6.

ISIS CANADA. Manual n.4: Strengthening Reinforced Concrete Structures with Externally-Bonded Fibre Reinforced Polymers (FRPs). Winnipeg, 2001.

JSCE. Japan Society of Civil Engineers. Recommendation for design and construction of concrete structures using continuous fiber reinforcing materials. Engineering Series 3. 1997.

JUNG, W. T.; PARK, J. S.; KANG, J. Y.; KEUM, M. S.. Flexural Behavior of Concrete Beam Strengthened by Near-Surface Mounted CFRP Reinforcement Using Equivalent Section Model. Advances in Materials Science and Engineering, Seul, v.2017, p.16, 2017.

KHALIFA, A. M.. Flexural performance of RC beams strengthened with near surface mounted CFRP strips. Alexandria Engineering Journal, Alexandria, v.55, p.14971505, 2016.

NORDIN, H.; TÄLJSTEN, B.. Concrete Beams Strengthened with Prestressed Near Surface Mounted CFRP. Journal of Composites for Construction, Lyngby, v.10, n1, p.60-68, 2006. 
PENG, H.; ZHANG, J.; CAI, C. S.; LIU, Y.. An experimental study on reinforced concrete beams strengthened with prestressed near surface mounted CFRP strips. Engineering Structures, Changsha, v.79, p.222-233, 2014.

REDA, R. M.; SHARAKY, I.A .; GHANEM, M.; SELEEM, M. H.; SALLAM, H. E. M.. Flexural behavior of RC beams strengthened by NSM GFRP Bars having different end conditions. Composite Structures, Ramadan City, v.147, p.131-142, 2016.

REZAZADEH, M.; COSTA, I.; BARROS, J.. Influence of prestress level on NSM CFRP laminates for the flexural strengthening of RC beams. Composite Structures, Guimarães, v.116, p.489-500, 2014.

SAID, H.. Deflection Prediction for FRP-Strengthened Concrete Beams. Journal of Composites for Construction, v.14, p.244-248, 2010.

SENA-CRUZ, J. M.; BARROS, J. A. O.; COELHO, M. R. F.; SILVA, L. F. F. T.. Efficiency of different techniques in flexural strengthening of RC beams under monotonic and fatigue loading. Construction and Building Materials, Guimarães, v.29, p. 75-182, 2011.

SHAHVERDI, M.; CZADERSKI, C.; MOTAVALLI, M.. Iron-based shape memory alloys for prestressed near-surface mounted strengthening of reinforced concrete beams. Construction and Building Materials, Dübendorf, v.112, p.28-38, 2016.

SHARAKY, I. A.; TORRES, L.; SALLAM, A.. Experimental and analytical investigation into the flexural performance of RC beams with partially and fully bonded NSM FRP bars/strips. Composite Structures, Girona, v.122, p.113-126, 2014.

SHARAKY, I. A.; TORRES, L.; COMAS, J.; BARRIS, C.. Flexural response of reinforced concrete $(R C)$ beams strengthened with near surface mounted (NSM) fibre reinforced polymer (FRP) bars. Composite Structures, Girona, v.109, p.8-22, 2013.
SHARAKY, I. A.; REDA, R. M.; GHANEM, M.; SELEEM, M. H.; SALLAM, H. E. M.. Experimental and numerical study of RC beams strengthened with bottom and side NSM GFRP bars having different end conditions. Construction and Building Materials, Zagazig, v.149, p.882-903, 2017.

SHUKRI, A. A.; HOSEN, M. A.; MUHAMAD, R.; JUMAAT, M. Z.. Behaviour of precracked $R C$ beams strengthened using the side-NSM technique. Construction and Building Materials, Kuala Lumpur, v.123, p.617-626, 2016.

SUN, Z. Y.; WU, G.; WU, Z. S.; LUO, Y. B.. Flexural strengthening of concrete beams with near-surface mounted steel-fiber-reinforced polymer composite bars. Journal of Reinforced Plastics, Nanjing, v.18, p.1529-1537, 2011.

TANG, W. C.; BALENDRAN, R. V.; NADEEM, A.; LEUNG, H. Y.. Flexural strengthening of reinforced lightweight polystyrene aggregate concrete beams with near-surface mounted GFRP bars. Building and Environment, Hong Kong, v.41, p.13811393, 2005.

TENG, J. G.; LORENZIS, L.; WANG, B.; LI, R.; WONG, T. N.; LAM, L.. Debonding Failures of RC Beams Strengthened with Near Surface Mounted CFRP Strips. Composites for Construction, Hong Kong, v.10, p.92-105, 2006.

WAHAB, N.; SOUDKI, K. A.; TOPPER, T.. Mechanism of Bond Behavior of Concrete Beams Strengthened with NearSurface-Mounted CFRP Rods. Journal of Composites for Construction, Waterloo, v.15, n.1, p.85-92, 2011.

WANG, B.; TENG , J. G.; LORENZIS, L.; ZHOU, L. M.; JINPING O.; JIN, W.; LAU, K. T.. Strain monitoring of RC members strengthened with smart NSM FRP bars. Construction and Building Materials, Hong Kong, v.23, p.1698-1711, 2008.

WU, G.; DONG, Z. Q.; WU, Z. S.; ZHANG, L. W.. Performance and Parametric Analysis of Flexural Strengthening for RC Beams with NSM-CFRP Bars. Journal of Composites for Construction, Nanjing, v.18, n.4, p.1-10, 2014.

A CBPC - Companhia Brasileira de Produção Científica (CNPJ: 11.221.422/0001-03) detém os direitos materiais desta publicação. Os direitos referem-se à publicação do trabalho em qualquer parte do mundo, incluindo os direitos às renovações, expansões e disseminações da contribuição, bem como outros direitos subsidiários. Todos os trabalhos publicados eletronicamente poderão posteriormente ser publicados em coletâneas impressas sob coordenação da Sustenere Publishing, da Companhia Brasileira de Produção Científica e seus parceiros autorizados. Os (as) autores (as) preservam os direitos autorais, mas não têm permissão para a publicação da contribuição em outro meio, impresso ou digital, em português ou em tradução. 